University of Zurich

Department of Economics

Working Paper Series

ISSN 1664-7041 (print)

ISSN 1664-705X (online)

Working Paper No. 230

\title{
Targeted Information and Limited Attention
}

\author{
Andreas Hefti and Shuo Liu
}

Revised version, December 2019 


\title{
Targeted Information and Limited Attention
}

\author{
Andreas Hefti and Shuo Liu*
}

December 2019

\begin{abstract}
We examine the implications of limited consumer attention for the targeting decisions of competing firms. Limited attention alters the strategic role of information provision as firms may become incentivized to behave as mass advertisers, despite perfect targeting abilities. We analyze the consequences of limited attention for targeting, strategic pricing, market shares, attention competition between firms, and the value of marketing data to firms. Accounting for limited attention in an otherwise standard targeting framework can explain several recent key issues from the advertising industry, such as consumer-side information overload or the increased usage of ad blocking tools.
\end{abstract}

Keywords: Targeted Advertising, Limited Attention, Ad Avoidance, Salience

JEL classification: D43; L13; M37

*Andreas Hefti: Department of Economics, University of Zurich, and School of Management and Law, Zurich University of Applied Science. Shuo Liu: Guanghua School of Management, Peking University. Emails: andreas.hefti@econ.uzh.ch, and shuo.liu@gsm.pku.edu.cn. We are grateful to Jean-Michel Benkert, Alessandro Bonatti, Andrea Canidio, Fabrizio Germano, Julia Grünseis, Igor Letina, Armin Schmutzler, Aleksei Smirnov, Philipp Strack, and seminar participants at the University of Zurich, Swiss IO Day 2015 (Bern), the EEA Annual Congress 2016 (Geneva), the EARIE Annual Conference 2016 (Lisbon), and the XXXI Jornadas de Economía Industrial (Palma) for discussions and useful suggestions. We thank John Christian and Danielle AdamsHausheer for proof-reading. We are also very thankful for the many helpful suggestions and critical remarks by two anonymous referees and the journal editor, Mark Armstrong. Shuo Liu acknowledges the financial support from the Key Laboratory of Mathematical Economics and Quantitative Finance (Peking University), the Ministry of Education of China. Andreas Hefti thanks Ines Brunner for her support. 


\section{Introduction}

Modern information systems, above all the Internet, allow sellers to gather an enormous amount of data about their potential customers. In principle, such data allows firms to target their ads towards precisely identified subsamples of consumers. Advertising research leaves little doubt that targeted information has become a major source of advertising revenue. Large businesses such as Axciom, IRI, and Nielsen earn their money by selling consumer data to individual companies. Sponsored search advertising, allowing firms to advertise to consumers who indicate an interest through their web search queries, has become "the largest source of revenues for search engines" (Ghose and Yang, 2009). According to the annual report by the Interactive Advertising Bureau (IAB), search advertising alone already has a steady $40 \%$ of total digital ad revenue, which was about $\$ 6.76$ billion in 2006 and nearly tripled to $\$ 18.81$ billion in 2014. Similarly, sponsored advertising has grown annually by around $30 \%$ from $\$ 1.12$ billion in 2011 to $\$ 1.88$ billion in 2013 , and is expected to grow further 1

Given the rich datasets about a firm's potential customers and the broad array of information sharing technologies, including email, SMS, tweeting and social networks, it may come as a surprise that several recent press releases have substantially challenged whether real-world targeting behavior really is beneficial for firms and consumers ${ }^{2}$ If the data about consumers is so comprehensive and detailed, and tailored advertising is better and cheaper as ever before ${ }^{3}$ why do so many consumers persistently complain about being overwhelmed by ads that seem to be of little relevance to them, while firms are apparently targeting their messages far less accurately than they could: ${ }^{4}$

In this article, we show that such puzzling observations can be explained by means of a stylized competition model of targeted information when the standard presumption of unlimited consumer attention is relaxed. Attention, as a central concept of cognition, has long been

\footnotetext{
${ }^{1}$ Data available online on www.iab.com/insights and www.emarketer.com. See Yao and Mela (2011) for similar facts on the importance of sponsored search in advertising, and Evans (2009) for a general survey on advertising data.

${ }^{2}$ Examples include the articles 'Does sponsored content work for advertisers?', Wall Street Journal, March 23, 2014 and 'Does targeting work?', The Ad Contrarian, Feb 01, 2012, and the IAB report 'Online consumers view and usage of ad blocking technologies', Sep 2014. Farahat (2013) shows that previous studies on targeted advertising may have largely overestimated its effectiveness due to not accounting for selection bias.

${ }^{3}$ See, e.g., 'How online advertisers read your mind', The Economist, Sep 2014.

${ }^{4}$ See, e.g., a practitioner's report posted on the website AdEspresso (available on www.adespresso.com/blog).
} 
investigated by psychology and neuroscience. Beginning with Miller (1956), there is extensive evidence that people have limited attention, meaning that they fail to recognize all the information they are exposed to once a certain threshold is reached. Given the superabundance of information stimuli generated by modern advertising, limited attention, therefore, seems a particularly relevant concept in a competition context. Indeed, the dominant and persistent preoccupation of the advertising industry that a seller's messages could simply be overlooked by the consumers they were intended for implies clearly that limited consumer attention is a critical factor in markets featuring targeted information. ${ }^{5}$

Main Results We study the effects of limited consumer attention in a targeting context by adopting a standard duopoly Hotelling framework with ex-ante uninformed consumers. Besides competing in prices, both firms need to choose which consumers to target with their messages, where targeting is a costly activity. Consumers, in turn, always choose their best perceived product. The standard (implicit) assumption of unlimited attention declares that consumers are always able to register and memorize any piece of information they are exposed to. By contrast, attention-constrained consumers may perceive only a subset of all the information available to them. Applied to our duopoly framework, the notion of limited attention means that consumers will perceive at most one of the firms when making their final purchase decisions.

Our main results, Theorems 1 and 2, show that consumer inattention has a profound impact on the equilibrium behavior of the firms. In particular, with attention-constrained consumers and sufficiently low information costs, firms strategically choose to behave as if they were mass advertisers, flooding the entire market with their messages, without considering consumer preferences in detail, and despite an infinitely precise targeting technology. This is antipodal to the standard prediction resulting from unlimited attention, where competition disciplines both firms to send their messages exclusively to their prime consumers (i.e., the consumers to whom the respective firm offers the most preferred option), even in presence of an arbitrarily small information cost. The driving force behind this discrepancy is that information targeted at prime consumers works as an efficient shield against wasteful business-stealing if and only if consumers pay attention to every piece of information they see. In other words, limited

\footnotetext{
${ }^{5}$ See, e.g., ' Advertising and technology $]$, The Economist, Sep 2014, Special Report.
} 
attention annihilates the protective character of information provision that would otherwise guide the firms' targeting decisions.

Our analysis further highlights some consequences of limited attention for strategic pricing, market shares and welfare. In general, traditional fundamentals, such as preferences or the degree of product differentiation, become less critical in determining equilibrium prices and market shares, as opposed to the ability of a firm to attract attention. Likewise, limited attention alters some of the conventional comparative statics of strategic pricing. For example, the standard positive correlation between the consumer-side transportation cost parameter and equilibrium prices (and profits) becomes negative with inattentive consumers. This reflects the fact that, with unlimited attention, the relevant equilibrium threat for a firm's pricing decision is that some consumers may switch to a competitor. By contrast, with limited attention the pricing decision is guided by the concern that some consumers may be priced out of the market. Regarding welfare, consumer inattention leads to inefficiency as firms do not utilize their targeting abilities in the mass advertising equilibrium, resulting in some consumers choosing dominated products. Moreover, the mass advertising equilibrium implies an additional consumer welfare loss because prices are higher compared to the full-attention benchmark.

Extensions We study several extensions of the baseline model. The essential effects of limited attention on equilibrium targeting remain the same throughout. Moreover, the variations provide additional insights. Specifically, in Section 5, we study a situation where firms need to compete actively for the limited attention of their targeted consumers. Firms can manipulate the likelihood of grabbing the attention of mutually targeted consumers by choosing how salient their messages should be. The resulting competition for attention endogenizes the perception chances of the firms and their costs of targeted information, and therefore also interferes with the targeting decisions themselves. We show that the information costs resulting from the strategic competition for attention must, nevertheless, always be such that mass advertising remains the unique equilibrium outcome. Moreover, in equilibrium both firms enter into competition for the attention of each consumer. These results may help to understand why the leading concern of modern advertising firms tenaciously remains whether their messages are even registered by 
consumers, while consumers persistently complain simultaneously about information overload and seemingly ill-targeted messages.

In Section 6 we incorporate into our analysis the possibility that consumers may choose to use ad blockers. Empirically, ad blocking has increased over time, and this has become a serious challenge for the advertising industry. Increased ad blocking rates may come as a surprise, particularly considering that targeting technology and marketing data about consumers have improved significantly. Our model confirms that such a development is indeed unexpected, at least while limited attention is unaccounted for. By contrast, limited attention implies that mass advertising remains an equilibrium outcome, and ad blocking is becoming a fairly robust equilibrium phenomenon, even with perfect targeting abilities.

The intuition for the above findings is as follows. Rational consumers choose to block incoming information if and only if the anticipated nuisance of processing this information exceeds the anticipated perceived value of the information provided. Accordingly, a consumer's ad blocking decision depends on the expected information structure of the targeting equilibrium. With unlimited attention, market forces discipline firms to target their ads to prime consumers as precisely as they can. In response, consumers, anticipating that i) they will be exposed to fewer ads, and ii) the received information is certain to be most relevant to them, will have fewer reasons to install ad blockers. Therefore, the efficient targeting equilibrium, where both firms target their prime segments exclusively, remains an equilibrium outcome with perfect targeting abilities, and no consumer blocks the incoming information. By contrast, with limited attention the consumer exposure to ads is not reduced even if the precision of the marketing data improves. This occurs because the targeting decisions by the firms are dominated by an intention to intrude their competitor's prime segment. It therefore follows that the firms remain locked in their roles as mass advertisers, despite having potentially perfect marketing data. Fearful that market forces may fail to guide them to their best options, nuisance-sensitive consumers are then inclined to use ad blockers to avoid the irritation caused by equilibrium information overload. 
Article Structure Section 2 reviews the related literature. Section 3 presents, and Section 4 analyzes the baseline model of targeted information. Sections 5 and 6 study the extensions to attention competition and ad blocking, respectively. Section 7 concludes. All proofs of formal claims can be found in the printed appendix. Finally, the following supplementary material can be found in the online appendix: i) a characterization of all targeting equilibria that arise by abstracting from price competition, ii) an extension to imperfect marketing data, iii) an analysis of the pricing-targeting game with a different time structure than in the main text, and iv) a discussion of persuasive advertising in the context of our model.

\section{Related Literature}

Our article primarily contributes to the literature on targeted advertising. Early articles on targeting consider homogeneous goods (Roy, 2000) or a monopoly market (Esteban et al. 2001). Subsequently, several articles study targeted advertising with differentiated products (e.g. Iyer et al., 2005; Esteban and Hernandez, 2007; Esteves and Resende, 2016). These articles (implicitly) assume that consumer attention is unbounded, implying that no rational firm would want to target consumers who are aware of more suitable products. Our article highlights the fact that this core feature of the targeting incentives vanishes with the introduction of attentionconstrained consumers. The resulting inefficient mass advertising equilibrium contrasts sharply with the segmenting property of equilibrium targeting as predicted by standard models.$^{6}$

In Brahim et al. (2011), firms need to choose their advertising intensities, i.e., the probabilities that the consumers receive their ads, alongside making their targeting decisions. Their notion of advertising intensity is not compatible with the concept of limited attention, because the success of a firm's advertising intensity is always invariant to the competitor's efforts. If attention is a scarce resource, the perception chances resulting from a battle for attention cannot be independent of each other, which becomes most evident in Section 5 of our article. More generally, the results in Brahim et al. (2011) are direct reflections of the targeting incentives

\footnotetext{
${ }^{6}$ If firms have no targeting abilities, e.g., as in Grossman and Shapiro (1984), then advertising to all consumers can be an equilibrium outcome with rational, ex-ante uninformed consumers, provided that advertising costs are low enough.
} 
given perfectly attentive consumers. With low targeting costs, each firm confines targeting to its prime segment, whereas both firms will target all consumers - but never at full intensity - if targeting costs are high. The latter follows because firms can benefit from targeting non-prime consumers only if these consumers miss out on a better option. High targeting costs generate such a situation simply because it is too expensive to advertise at full intensity to one's prime segment. Limited attention produces an opposing prediction; mass advertising is the unique equilibrium with low targeting costs (or endogenous attention competition).

Van Zandt (2004) considers a model of targeted information with non-competing products, where consumers are only able to sample a subset of products in their information set with an exogenously given and uniformly distributed probability. By contrast, we study the case of competitive products with the possibility of non-uniform or endogenous perception chances, introduce price and salience competition as well as ad blocking to the model, and compare the limited attention prediction to its standard counterpart.

We also contribute to the literature on ad blocking (e.g., Armstrong et al., 2009; Anderson and Gans, 2011; Gritckevich et al., 2018), by studying a variant of ad blocking closely related to Johnson (2013). The main argument is that limited attention leads to the mass advertising equilibrium jointly with a number of blocking consumers, despite perfect targeting abilities, where such an equilibrium could not occur in the case of unlimited attention.

More broadly, our article contributes to the growing literature on behavioral industrial organization. Articles in this field are interested in how market outcomes are determined by the interaction between boundedly rational consumers and fully rational, profit-maximizing firms. Ellison (2006), Spiegler (2011), and Heidhues and Köszegi (2018) provide comprehensive surveys on the topic.

\section{A Model of Targeted Information}

Consider two firms, indexed by $j \in\{A, B\}$, located on a standard Hotelling line $[0,1]$. Let $x_{j}$ denote the location of firm $j$, where $x_{A}=0$ and $x_{B}=1$. A unit mass of consumers is uniformly distributed over the line. If a consumer $i \in[0,1]$ transacts with firm $j$, she receives a benefit 
$U_{i}(j)=V-p_{j}-t\left|i-x_{j}\right|$, where $V>0$ is the maximal willingness-to-pay, $p_{j} \geq 0$ is the price of product $j$, and $t\left|i-x_{j}\right|, t>0$, quantifies the consumer-side (transportation) costs. Let $\mathcal{P}_{j} \equiv\left\{i: U_{i}(j) \geq U_{i}(-j)\right\}$ denote firm $j$ 's prime segment of consumers. If $\left|p_{B}-p_{A}\right|<t$, then $\mathcal{P}_{A}=\left[0, i_{0}\right]$ and $\mathcal{P}_{B}=\left[i_{0}, 1\right]$, where the indifferent consumer $i_{0} \in(0,1)$ has location

$$
i_{0}=\frac{p_{B}-p_{A}}{2 t}+\frac{1}{2}
$$

If $\left|p_{B}-p_{A}\right| \geq t$, the prime segment of one firm coincides with the entire consumer population.

Information, Attention, and Consumer Choice Most of the literature in psychology and neuroscience depicts attention as the critical cognitive gating mechanism selecting which subset of the available information is perceived by a decision-maker in case of information overload (see Hefti and Heinke, 2015; Hefti, 2018). Our main contribution is to embed limited consumer attention into an otherwise standard targeting setting defined on the above Hotelling framework. We introduce limited attention by assuming that each consumer $i$ is endowed with an attention capacity $R_{i} \in\{1,2\}$, which determines the maximal number of alternatives that can be perceived $]^{7}$ Let $X_{i} \subseteq\{A, B\}$ denote consumer $i$ 's information set, recording the firms from which $i$ has received information. Then, $A_{i} \subseteq X_{i}$ denotes $i$ 's attention set, i.e., the subset of alternatives that $i$ bases her decision on. Concerning choice, a consumer $i$ always transacts with her best perceived firm if $A_{i} \neq \emptyset$ and her net utility is non-negative, and not at all otherwise.

Standard economic models do not need to differentiate between $A_{i}$ and $X_{i}$ given their (implicit) assumption that attention is unconstrained. However, the received and perceived information may differ for a consumer with limited attention: If $R_{i}=1$ then $A_{i}=X_{i}$ if and only if $X_{i} \subsetneq\{A, B\}$. Further, if $R_{i}=1$ attention selects which firm is perceived in case of information overload $\left.\left(\left|X_{i}\right|>1\right)\right]^{8}$ In particular, the probability $P\left(j \in A_{i}\right)$ that firm $j \in\{A, B\}$

${ }^{7}$ Limited attention as a capacity threshold on the number of distinguishable objects has also been used, e.g., by Van Zandt (2004) or Anderson and De Palma (2009, 2012). Other approaches to limited attention invoke a volume-based threshold (Falkinger, 2007) or a threshold on the processable amount of bits (Sims, 2003).

${ }^{8}$ We do not have in mind that $R_{i}=1$ necessarily means that a consumer perceives only a single product, but instead only one menu of products, like an IKEA catalog, in which case $j$ is the underlying brand. Nevertheless, in a recent study using sales data from Amazon's Electronics category, Gu et al. (2012) find that more than 
is perceived is determined by

$$
P\left(j \in A_{i}\right)= \begin{cases}1 & \text { if } j \in X_{i} \text { and }\left|X_{i}\right|=1, \\ \pi_{j} & \text { if } j \in X_{i} \text { and }\left|X_{i}\right|=2, \\ 0 & j \notin X_{i} .\end{cases}
$$

The salience parameters $\pi_{A}, \pi_{B} \in(0,1)$ satisfy $\pi_{A}+\pi_{B}=1$. Note that if $R_{i}=2$, then $P\left(j \in A_{i}\right)=1$ if and only if $j \in X_{i}$. This innocent-looking equivalence between information and perception causes a diametrically different characteristic of the equilibrium targeting behavior compared to its counterpart with limited attention. In the main analysis this is elucidated by comparing the equilibria for the two polar cases, where either all consumers have unlimited attention $\left(R_{i}=2 \forall i\right)$, or all consumers have limited attention $\left(R_{i}=1 \forall i\right) !^{9}$

Targeting To make the effects of consumer (in)attention on firm-side targeting most evident, we will suppose that firms are endowed with perfect information about each consumer's location, meaning that they possess perfect targeting abilities. ${ }^{10}$ A targeting strategy is an indicator function $g_{j}:[0,1] \rightarrow\{0,1\}$, where $g_{j}(i)=1$ means that consumer $i$ is targeted by firm $j$. Let $\mathcal{L}$ be the set of all measurable indicator functions on $[0,1]$, and $\left(g_{A}, g_{B}\right) \in \mathcal{L}^{2}$ is a targeting profile. Further, $\mathcal{I}_{j}=\left\{i: g_{j}(i)=1\right\}$ is the set of consumers targeted by firm $j$. Let $\lambda(S)$ denote the (Lebesgue) measure of a measurable set $S \subseteq[0,1]$. Each firm's expenditure for its information campaign is $c \lambda\left(\mathcal{I}_{j}\right)$, where $c>0$ is the marginal information cost. This is a standard cost function in the targeting literature (see, e.g., Van Zandt, 2004; Iyer et al., 2005; Bergemann and Bonatti, 2011; Johnson, 2013).11 The following two properties of a targeting profile are relevant for our analysis.

\footnotetext{
$78 \%$ of consumers consider only a single product at the time of purchase.

${ }^{9}$ The mixed case where a fraction $q \in[0,1]$ of the consumers have limited attention, while $1-q$ of them have unlimited attention, is analyzed in the online appendix.

${ }^{10}$ This is relaxed in the online appendix (see Propositions B1 and B2).

${ }^{11}$ We later consider "pay-per-click" costs as an alternative cost structure.
} 
Definition 1 A targeting profile is intrusive if at least one firm uses an intrusive targeting strategy, that is, $\exists j \in\{A, B\}$ such that $\lambda\left(\mathcal{I}_{j} \cap \mathcal{P}_{-j}\right)>0$. A targeting profile is overlapping if $\lambda\left(\mathcal{I}_{A} \cap \mathcal{I}_{B}\right)>0$.

A targeting profile thus is intrusive if at least one firm targets non-prime consumers, and it is overlapping if it features jointly targeted consumers. Overlapping targeting is sufficient but not necessary for intrusive targeting given our definition of prime segments.

Pricing-Targeting Game In a digital economy, it is perfectly conceivable that firms have prior knowledge about their rivals' pricing strategies when launching their advertising campaigns. For example, although iPhone7 was not yet available at the time this article was first drafted, its price had already been leaked on several websites ${ }^{12}$ and such information would almost certainly have been spotted by the competitors of Apple (if they did not know it already). A related common observation is that once prices are set, firms may be reluctant to change them, which is a well-known outcome in the presence of menu costs (see, e.g., Golosov and Lucas, 2007). Moreover, most advertising has become digital, predominantly featuring realtime auctions for ad placement in digital ad space, which essentially means that advertising can be adjusted instantaneously ${ }^{13}$ This is substantially different from traditional, non-digital advertising, such as the design of a TV spot or a printed poster, which cannot be easily altered in the short run.

Motivated by the above reasons, we study a two-stage complete information game, where both firms first set their prices, and then decide on which subset of consumers to target. As these pricing decisions determine the prime segments $\mathcal{P}_{A}, \mathcal{P}_{B}$, this two-stage structure has the additional upshot of making most evident how consumer (in-)attention affects equilibrium targeting ${ }^{14}$ In the online appendix, we show that limited attention has the same consequences for equilibrium targeting if firms decide on prices and targeting simultaneously.

\footnotetext{
${ }^{12}$ See, e.g., http://bgr.com/2016/06/30/this-is-the-iphone-7-leak-weve-been-waiting-for.

${ }^{13}$ See the 'AdEX Benchmark H1 2017 Study' published by IAB Europe. A related video illustration is available at https://goo.gl/YcUjJy.

${ }^{14}$ This also is the essential type of timing underlying the targeting model of Brahim et al. (2011).
} 


\section{Equilibrium Analysis}

Consumer attention is decisive for the nature of the targeting incentives. To see this, fix a firm $j \in\{A, B\}$, and consider any consumer $i \in \mathcal{P}_{j}$ with $U_{i}(j) \geq 0$. If consumer attention is unlimited, then targeting $i$ is necessary and sufficient for firm $j$ to transact with $i$. It follows that whenever $p_{j}>c, j$ has a strict incentive to target $i$, independent of its competitor's behavior, whereas $-j$ will never choose to target $i$. By contrast, with limited attention there is a chance that the messages sent by firm $-j$ manage to inhibit the perception of firm $j$. Therefore, firm $-j$ rationally chooses to target $i$ if the expected revenue from attention exceeds information costs $\left(\pi_{-j} p_{-j}>c\right)$, independent of $j$ 's targeting behavior. If also $\pi_{j} p_{j}>c$, then both firms have an incentive to target the consumer, despite $i \in \mathcal{P}_{j}$. This simple insight about how limited attention alters the targeting incentives is critical to all our principal findings.

Targeting Equilibrium We first derive the effects of limited attention on equilibrium targeting for the case where prices, and hence prime segments, are exogenously given. In terms of the pricing-targeting game, this corresponds to a particular second-stage targeting subgame. More generally, the following analysis connects to several contributions on targeting, such as Van Zandt (2004), Athey and Gans (2010) and Johnson (2013), all of which have abstracted from price competition. In addition, the setting with exogenous prime segments also accommodates other interpretations of our model. For example, the prime segments could be defined by (Downsian) voter preferences in an election with two different political candidates, who must decide which voters to target.

Fix $p_{A}, p_{B} \in(c, V-t]$, and suppose that firms simultaneously choose their targeting strategies to maximize expected payoffs. A targeting equilibrium is a targeting profile such that neither firm can gain a strict advantage by deviating to any alternative targeting strategy. Further, we call a targeting equilibrium unique if it is unique in the measure $\lambda$ of targeted consumers it induces; any two equilibria only differ by zero-measure sets ${ }^{15}$

In the standard case of unlimited attention, it readily follows from the targeting incen-

\footnotetext{
${ }^{15}$ As we allow for general Lebesgue-measurable targeting functions, uniqueness of equilibrium cannot be obtained in the narrow sense.
} 
tives previously outlined that the unique targeting equilibrium is characterized by both firms exclusively targeting their prime segments. In particular, equilibrium targeting can never be intrusive (hence never overlapping), even if the information cost $c$ is arbitrarily small: Firm $j$ would only send its messages to a non-prime consumer $i$, if $i$ is not targeted by $-j$ or if $j$ 's messages could inhibit the perception of the ones sent to $i$ by $-j$. With unlimited attention and rational firms, this situation cannot occur. The following proposition summarizes this insight.

Proposition 1 (Segmentation) The targeting game with unlimited attention has a unique equilibrium. In equilibrium, $\lambda\left(\mathcal{I}_{j} \cap \mathcal{P}_{-j}\right)=0$ and $\lambda\left(\mathcal{I}_{j} \cap \mathcal{P}_{j}\right)=\lambda\left(\mathcal{P}_{j}\right), \forall j \in\{A, B\}$.

A natural targeting equilibrium is given by the "interval strategies" $g_{A}(i)=1$ iff $i \in \mathcal{P}_{A}$, and $g_{B}(i)=1$ iff $i \in \mathcal{P}_{B}$. Recalling our measure-theoretic notion of uniqueness, any other equilibrium differs from these interval strategies only by zero-measure sets.

The segmenting nature of equilibrium targeting strategies identified by Proposition1 1 strongly resonates with the targeting literature (see, e.g., Iyer et al., 2005; Esteban and Hernandez, 2007; Brahim et al., 2011; Esteves and Resende, 2016). We now demonstrate that this type of targeting behavior is driven by the assumption of unlimited attention. In particular, the next result shows that with limited attention and sufficiently low information costs, the only equilibrium outcome is characterized by firms behaving as if they were mass advertisers, despite their perfect targeting abilities.

Proposition 2 (Mass Advertising) The targeting game with limited attention and $c<\pi_{j} p_{j}$, $j \in\{A, B\}$, has a unique equilibrium, and $\lambda\left(\mathcal{I}_{j}\right)=1, j \in\{A, B\}$.

The fact that both firms choose to target the entire market, independent of their prime segments, is again a direct consequence of the targeting incentives induced by limited attention. It reflects the fact that limited attention annihilates the protective character of information provision, which would otherwise discipline firms to abstain from intrusive targeting. 
In the mass-advertising equilibrium, the following implications of limited attention for the determination of firm market shares and welfare emerge. First, market shares $m_{j} \in[0,1]$ and firm profits $\Pi_{j}$ precisely reflect the size of each firm's prime segment with unlimited attention, because then $m_{j}=\lambda\left(\mathcal{P}_{j}\right)$ and $\Pi_{j}=\left(p_{j}-c\right) m_{j}$. By contrast, $m_{j}=\pi_{j}$ and $\Pi_{j}=\pi_{j} p_{j}-c$ for limited attention. In other words, market shares and profits are invariant to prime segments and determined only by the ability to attract attention. At its extreme, this means that a comparative advantage in attention-seeking may crowd out an otherwise superior firm. ${ }^{16}$ Second, as information is costly, efficiency requires that each firm exclusively targets its prime segment; an intrusive targeting profile is inefficient. By Proposition 1, the market forces could always be relied upon to induce the informationally efficient outcome in case of unlimited attention. By contrast, with limited attention the market forces lead to wasteful targeting in the mass advertising equilibrium identified in Proposition 2.

In the online appendix, we generalize Propositions 1 and 2 in several directions. In particular, we allow for firm heterogeneity concerning product differentiation and information costs, as well as for consumer heterogeneity in $R_{i}$. The general analysis reveals that a locational structure, like Hotelling, is not crucial for our main results; all that is needed is a measurable assignment of consumers to prime segments. In addition, we derive the targeting equilibria for parameter constellations that differ from those in Propositions 1 and 2. If, contrary to Proposition 2, information costs are sufficiently high, the firms are engaged in a coordination game featuring a continuum of intrusive but always non-overlapping targeting equilibria. These equilibria arise once the information expenditures for mutually targeted consumers are not covered anymore. However, the coordination equilibria are not robust; for example, they cannot arise with an endogenous information cost due to salience competition (see Section 5).

Pricing-Targeting Equilibrium We now analyze the subgame perfect equilibria (SPE) of the pricing-targeting game. Each firm's strategy is $\left(p_{j}, g_{j}\right)$, where $p_{j} \in \mathbb{R}_{+}$is the first-stage price and $g_{j}$ is the second-stage targeting function. We impose the standard full-coverage assumption $V-2 t>c$ assuring that a monopolist located at $x \in\{0,1\}$ would choose to serve the entire

\footnotetext{
${ }^{16}$ See Section 1 in the online appendix for a more formal discussion.
} 
market at price $p^{M}=V-t$. The first result characterizes the unique SPE outcome in the case of unlimited attention.

Theorem 1 (Unlimited Attention) In the unique SPE of the equilibrium pricing-targeting game with unlimited attention, the targeting profile splits the market according to prime segments, i.e., $\mathcal{I}_{A}=\mathcal{P}_{A}=[0,1 / 2]$ and $\mathcal{I}_{B}=\mathcal{P}_{B}=[1 / 2,1]$. Equilibrium prices are $p_{A}^{*}=p_{B}^{*}=t+c$. Theorem 1 confirms that with unlimited attention and endogenous prices, only a non-intrusive targeting equilibrium results. In particular, targeting strategies by firms exactly match the respective prime segments induced by the prices chosen in the first stage. The intuition for this central property of the SPE follows directly from the disciplining nature of information provision caused by unlimited attention, as outlined in the previous section.

Further, Theorem 1 shows that unlimited attention also restrains strategic pricing. It can be verified that the prices in the SPE of Theorem 1 correspond exactly to their counterparts in the traditional Hotelling setting where consumers are endowed with complete information, and $c$ has the conventional interpretation of a production cost (d'Aspremont et al., 1979). This type of equivalence is not coincidental and holds much more generally, as we prove in the Appendix. The main intuition is as follows: First, optimality requires that the targeting strategies match the respective prime segments. As a consequence, the only market segmentation that can be sustained on the equilibrium path is the one following the complete information prices $p_{j}^{*}=t+c$. Any other prices would induce a deviation incentive for at least one firm, as consumers with unlimited attention behave like perfectly informed consumers when targeted by both firms. For instance, if $p_{A}>p_{B}=t+c$, then firm $A$ has a strict incentive to lower its price and target accordingly, as this price deviation would also be profitable in the case of perfectly informed consumers.

The above equilibrium structure collapses with limited attention. As the next theorem shows, limited attention is a source of market power regarding product pricing, which complements the incentives to act as mass advertisers. 
Theorem 2 (Limited Attention) If $c<\pi_{j}(V-2 t), \forall j \in\{A, B\}$, then the pricing-targeting game with limited attention has a unique SPE, where both firms behave as mass advertisers and charge the monopoly prices, i.e., $\lambda\left(\mathcal{I}_{A}\right)=\lambda\left(\mathcal{I}_{B}\right)=1$ and $p_{A}^{*}=p_{B}^{*}=V-t$.

In the proof of Theorem 2 we establish that neither the symmetry of firm locations nor the locational structure is critical for mass advertising to emerge as part of an SPE with limited attention. The reason behind the mass advertising strategy is that limited attention makes the prime segments irrelevant for optimal targeting. Accordingly, the main concern guiding a firm's pricing decision is that a consumer targeted by that firm finds the product sufficiently attractive relative to the outside option. The optimal price, therefore, corresponds to the full-coverage monopoly price $p^{*}=V-t$.

A comparison of Theorems 1 and 2 further reveals that limited attention has additional implications for how consumer characteristics influence the respective equilibrium with endogenous pricing. Most notably, equilibrium prices and profits increase in the taste parameter $t$ with attentive consumers but decrease in $t$ with inattentive ones. The reason is that $t$ affects both the willingness-to-substitute and the decisions about whether or not to acquire a product at all. With unlimited attention, the main threat for a firm is losing consumers to its competitor, which makes product substitutability crucial for strategic pricing. As this substitutability depends negatively on $t$, it follows that a lower value of $t$ reduces the pricing power for each firm. Conversely, if attention is limited, only the threat of losing consumers matters to firms. A higher $t$ increases this threat, forcing firms to introduce lower prices.

Regarding welfare, the SPE is efficient with unlimited attention, and inefficient otherwise. More specifically, with unlimited attention, the competitive forces annihilate any excessive targeting, and the induced segmentation of the market is efficient given consumer preferences. By contrast, the mass advertising equilibrium in Theorem 2 is inefficient because it features wasteful targeting, and some consumers end up acquiring a dominated product. Moreover, limited attention implies an additional loss in consumer welfare with endogenous pricing, as consumers are charged comparably higher prices ${ }^{17}$

\footnotetext{
${ }^{17}$ In a previous working paper version, we provided a quantitative welfare analysis with non-extreme firm locations. The welfare loss due to limited attention becomes particularly pronounced the more firms are differ-
} 
Pay-Per-Click Costs So far, our analysis has been based on the standard cost structure in the advertising literature, which assumes that firms pay a fixed piece-rate per targeted consumer, independent of whether a successful transaction results. Recently, performancebased pricing schemes, to which "pay-per-click" prominently belongs, have gained importance among implemented real-world pricing structures ( $\mathrm{Hu}$ et al., 2015). It is straightforward to verify that the equilibrium effects of limited attention remain unchanged if the previous "payper-impression" is replaced by a "pay-per-click" scheme. Suppose that firm $j$ incurs the unit cost $c>0$ for its targeted information if and only if it receives a click by a consumer, and consumers click only on as many ads as they can cognitively process ${ }^{18}$ Then, the expected net payoff from a mutually targeted consumer is $\pi_{j}\left(p_{j}-c\right)$, as opposed to $\pi_{j} p_{j}-c$ in the previous analysis. Thus, targeting tends to become less expensive under pay-per-click. This further strengthens the incentive for firms to behave as mass advertisers in case of limited attention. For the unique equilibrium outcome in Theorem 2 to arise, the full coverage assumption $c<V-2 t$ suffices. ${ }^{19}$

\section{Attention Competition}

There is a large amount of evidence suggesting that how much attention firms manage to attract depends on the conspicuousness or salience of their messages relative to those of others (Hefti and Heinke, 2015). In reality, efforts to attract or maintain attention take on many forms, such as advertising on multiple channels (e-mails, social networks, phone calls, etc.) to a consumer or re-targeting consumers who have demonstrated previous interest. How does strategic targeting evolve if firms need to compete actively for the attention of their consumers?

We provide an answer to this question by enriching the targeting framework with firm-side attention competition as in Hefti (2018). Each firm now must decide how much to invest in the salience of its messages, alongside choosing its pricing and targeting strategies. Formally, each firm's strategy in the targeting stage is a pair $\left(g_{j}, s_{j}\right)$, where $s_{j}:[0,1] \rightarrow \mathbb{R}_{+}$is the salience

\footnotetext{
entiated from each other.

${ }^{18}$ If attention-constrained consumers click on all the ads they see but remember only one firm when making their consumption decisions, the relevant information cost and the strategic analysis, would be identical to the previous case of pay-per-impression.

${ }^{19}$ In the case of unlimited attention, consumers click on all ads they receive. This yields the same effective costs as with pay-per-impression, and therefore results in the segmenting equilibrium identified by Theorem 1.
} 
function, which specifies the salience level $s_{j}(i)$ at which each consumer $i$ is targeted. The chosen salience levels endogenize the perception chances $\left(\pi_{A i}, \pi_{B i}\right)$ by an inattentive consumer $i$ according to the following principle: If $i$ is targeted by one firm only, this firm is always perceived for any given salience level; if $i$ is targeted by both firms, a contest for attention emerges, and the perception chances are determined by relative salience:

$$
\pi_{j i}= \begin{cases}\frac{s_{j}(i)}{s_{A}(i)+s_{B}(i)} & \text { if } s_{A}(i)+s_{B}(i)>0 \\ \frac{1}{2} & \text { otherwise. }\end{cases}
$$

Salience-generating activities thus increase your own and decrease your competitor's perception chances. We suppose that targeting a message to a consumer at a salience level $s$ requires the firm to incur a cost of $h(s)$, where $h: \mathbb{R}_{+} \rightarrow \mathbb{R}_{+}$is a convex and strictly increasing $C^{2}$-function with $h(0)=0$. Accordingly, for a given information campaign $\left(g_{j}, s_{j}\right)$, firm $j$ 's information expenditure is $\int_{[0,1]} h\left(s_{j}(i)\right) d i$. Hence, salience competition also endogenizes the marginal information costs ${ }^{20}$ As salience competition emerges only with limited attention, the following analysis is confined to this case.

Targeting-Salience Equilibrium To make the effects of salience competition on firms' targeting decisions most evident, we first take $p_{A}=p_{B}=p \in(0, V-t]$ as exogenously fixed. Given the two-stage game with endogenous prices, this corresponds to a particular secondstage subgame. The key insight is that, in equilibrium, the marginal information costs will always be sufficiently low, relative to the price $p$, such that the cost condition in Proposition 2 is endogenously satisfied, which incentivizes the firms to act as mass advertisers.

Proposition 3 In the unique equilibrium of the above targeting-salience game both firms behave like mass advertisers, i.e., $\lambda\left(\mathcal{I}_{A}\right)=\lambda\left(\mathcal{I}_{B}\right)=1$, and set the same salience level $s^{*}>0$ for each consumer, where $s^{*}$ uniquely solves $p=4 h^{\prime}\left(s^{*}\right) s^{*}$.

\footnotetext{
${ }^{20}$ Recall that in the baseline model, firm $j$ 's total information cost is given by $c \int_{[0,1]} g_{j}(i) d i$, where $c>0$ is exogenous. This can be viewed as a special case of the current model where firm $j$ is exogenously given the following salience function: $s_{j}(i)=0$ if $g_{j}(i)=0$ and $s_{j}(i)=h^{-1}(c)$ otherwise.
} 
The intuition is as follows. There cannot be a positive measure set of untargeted consumers in equilibrium because each firm would find it profitable to target such consumers at the minimum salience level. Now, consider a jointly targeted consumer $i$, where firm $-j$ chooses a salience level $s_{-j}(i)>0$. Then, firm $j$ 's optimal salience level is determined by the first-order condition

$$
p \frac{s_{-j}(i)}{\left(s_{j}(i)+s_{-j}(i)\right)^{2}}=h^{\prime}\left(s_{j}(i)\right) \text {. }
$$

The equilibrium system implied by (3) only has a symmetric solution $s_{A}(i)=s_{B}(i)=s>0$. Hence (3) reduces to $p=4 h^{\prime}(s) s$ in any equilibrium. Then, mass advertising is the unique equilibrium targeting outcome because the expected revenues from any jointly targeted consumer must exceed the respective salience expenditures as a consequence of the convexity of $h(\cdot)$.

The result in Proposition 3 is of practical interest. The leading concern of modern advertising firms is whether their messages are actually registered by consumers. ${ }^{21}$ In reality, a sizable sum of money is invested in remaining "top of the mind", despite the availability of sophisticated marketing data, and the fact that products may already be familiar to many consumers (Iyer et al., 2005). Such an empirical regularity is hard to rationalize with perfectly attentive consumers - salience competition would not occur even with weak marketing data about consumer preferences. By contrast, Proposition 3 shows that intense advertising for attention jointly with seemingly ill-targeted messages is a well-anticipated pattern if consumers have limited attention.

SPE with Endogenous Pricing We now analyze the full-fledged model, where firms first choose prices, and then simultaneously decide on their targeting and salience functions. For tractability only, we assume that $h(s)=s$. The following result shows that if $V / t$ is sufficiently large, the targeting-salience equilibrium in Proposition 3 emerges as part of a unique SPE.

Theorem $3 A$ unique SPE in the pricing-targeting-salience game exists if $V / t$ is sufficiently large (in particular, if $V / t \geq 5.9$ ). In equilibrium, both firms behave like mass advertisers,

\footnotetext{
${ }^{21}$ See, e.g., 'Invisible ads, phantom readers', The Economist, March 2016.
} 
choose the same salience level $s^{*}=\frac{V-t}{4}$ for all consumers, and charge the monopoly price, i.e., $p_{A}^{*}=p_{B}^{*}=V-t$.

The intuition for the second-stage mass advertising is the same as in Proposition 3. Likewise, the equilibrium salience level corresponds to the one in Proposition 3 given that $p=V-t$ and $h(s)=s$. The condition $V / t \geq 5.9$, which is sufficient but necessary, assures that the cutoff monopoly price is sufficiently attractive such that no firm wishes to deviate to a higher price at risk of losing some consumers to the outside option. Further, the proof of Theorem 3 reveals that mass advertising also occurs with asymmetric firm locations. Here, firms may choose different salience levels in equilibrium, as the advantaged firm has a higher incentive to attract consumer attention given its higher monopoly price.

\section{Information Blocking and Limited Attention}

In recent years, the advertising industry has faced the increasing use of digital ad blocking tools, despite improved targeting abilities and virtually unchanged blocking technologies ${ }^{22}$ According to the report 'The cost of ad blocking'' by PageFair and Adobe (2015) the year 2014 saw a $48 \%$ increase in ad blocking in the US; the estimated revenue loss to the industry is $\$ 21$ billion, or $14 \%$ of global ad spending. The IAB also reported that more than one-third of US adults use ad blockers.

This development caught the ad industry by surprise, which is understandable given how targeting is supposed to evolve, at least as long as limited attention is not accounted for. With strong targeting abilities, perfect consumer attention would force firms to focus their advertising exclusively on their respective prime segments. Consumers, in turn, would anticipate that the market provided them solely with the most suitable information, thus making them hesitant to use ad blockers. By contrast, the mass advertising equilibrium resulting from limited attention implies that consumers cannot rely on market forces to guide them to their best options. Consequently, consumers who expect to be flooded by information of varying degrees of usefulness

\footnotetext{
${ }^{22}$ See 'Does targeted advertising work in 2015 ', and 'ClarityRay battles ad blockers with $\$ 500 \mathrm{~K}$ in funding]', May 2012. For company advice how to respond to increased ad blocking, see 'Ad blocking: the unnecessary Internet apocalypse', Sep 2015.
} 
may become inclined to block all incoming ads. Indeed, several inquiries concluded that the recent increase in ad exposure, leading to a sense of information overload by consumers, is a major reason why people started using ad blocking software. ${ }^{23}$

We make the above intuitive inattention-based explanation for increased ad blocking precise by incorporating the consumer decision whether or not to block incoming information into our two-stage, pricing-targeting framework from Section 3. Each consumer $i$ makes a blocking decision $b_{i} \in\{0,1\}$ simultaneous to the firms' choices of targeting strategies. If $b_{i}=0$, the consumer does not block and decides as in the baseline model; if $b_{i}=1$, any information received is blocked before inspection, and $i$ earns the reservation utility zero. Following Johnson (2013), each consumer $i$ bears a nuisance cost $\gamma_{i} \in[0, \bar{\gamma}]$ for each incoming message, where $\gamma_{i}$ is private information, and an iid draw from a known distribution $F(\cdot)$. As in the baseline model, consumers are ex-ante uninformed about their locations on the Hotelling line. That is, in the absence of any incoming messages, they only hold the (correct) prior belief about their match values with the two firms. Thus, consumers face a trade-off between enduring ads and forgoing consumption utility, and reach their blocking decisions by forming rational expectations about the value of the incoming information. The expected net utility of listening depends on the anticipated amount of incoming information, the nuisance cost and the anticipated match between their tastes and the perceived information. We impose the tie-breaking rule that a consumer does not block when indifferent, which excludes the trivial equilibrium where all consumers block, and both firms target a zero-measure set of consumers.

We first show that only non-intrusive targeting profiles can be sustained in equilibrium with unlimited attention and perfect targeting abilities. In particular, mass advertising cannot arise.

Theorem 4 In any equilibrium of the pricing-targeting-blocking game with unlimited attention, the targeting profile is non-intrusive, i.e., $\lambda\left(\mathcal{I}_{j} \cap \mathcal{P}_{-j}\right)=0, j \in\{A, B\}$. Moreover, the situation where targeting sets are $\mathcal{I}_{A}=\mathcal{P}_{A}=[0,1 / 2], \mathcal{I}_{B}=\mathcal{P}_{B}=[1 / 2,1]$, prices are $p_{A}=p_{B}=t+c$, and no consumer blocks information, is an equilibrium whenever $\bar{\gamma} \leq V-5 t / 4-c$.

\footnotetext{
${ }^{23}$ See 'Online consumers views and usage of ad blocking technologies', IAB report, Sep 2014., or 'B2B ad blocker study of the OVK', Oct 2015. Also see 'Invisible ads, phantom readers', The Economist, March 2016.
} 
The main finding based on Theorem 4 is that, as before, unlimited consumer attention disciplines firms against intrusive targeting, even with potentially blocking consumers. The intuition is as follows. Firm $j$ would only have an incentive to intrude if enough consumers decided not to block and $-j$ fails to target all consumers in its prime segment. Such an omission is only rational for firm $-j$ if it cannot benefit from targeting these prime consumers, which implies that it must be making non-positive profits with them. However, because $j$ must earn a positive profit from these consumers, the pricing of $-j$ cannot have been optimal. Moreover, if both firms target their prime segments exclusively, rational consumers anticipate that they will receive only the most valuable information in the market. ${ }^{24}$ The assumption $\bar{\gamma} \leq V-5 t / 4-c$ then assures that whenever consumers expect $p \leq t+c$, blocking will not arise. However, given that consumers do not block, both firms will indeed target only their prime segments at a price $p=t+c$, consistent with consumer expectations ${ }^{25}$

We now analyze the same situation for limited attention. For simplicity, we take the perception probabilities as given by $\pi_{A}=\pi_{B}=1 / 2$.

Theorem 5 Suppose that $c<\frac{F(\hat{\gamma})}{2}(V-2 t)$, where $\hat{\gamma}=\frac{t}{4}$. The pricing-targeting-blocking game with limited attention has an equilibrium where both firms behave as mass advertisers and charge the monopoly price, i.e., $\lambda\left(\mathcal{I}_{j}\right)=1$ and $p_{j}=V-t \forall j \in\{A, B\}$, and a fraction $1-F(\hat{\gamma})$ of consumers block. If, in addition, $F(\hat{\gamma})<1$, then every equilibrium features consumer blocking. As before, limited attention together with low information costs induces an incentive for the firms to target the entire market, independent of their prime segments. If both firms adopt such a strategy, consumers without ad blockers will be flooded with information of sometimes low relevance, and may end up with a dominated alternative. Anticipating such a possibility, consumers with sufficiently high nuisance costs rationally choose to block. Hence, with low enough information costs, both firms remain locked in their roles as mass advertisers, despite

\footnotetext{
${ }^{24}$ The expected utility (net of prices and nuisance costs) from choosing the better option if a consumer expects to receive information from both firms is $V-t / 4$.

${ }^{25}$ Theorem 4 reflects a result in Johnson (2013) who finds, in a different setting, that blocking vanishes if targeting abilities become accurate. In his model, blocking is a direct consequence of imperfect marketing data, and reflects the fact that some consumers receive pure spam as a consequence of targeting inaccuracy.
} 
possibly losing substantial sales to blocking consumers. This is in sharp contrast to the nonintrusive targeting profile implied by unlimited attention. Theorem 5 further shows that, with limited attention, a blocking-free equilibrium is not generally attainable. The reason for this is that whenever all consumers choose not to block, it is optimal for firms to target the entire market at the monopoly price. If nuisance costs are not negligible (in particular, they are not always less than $t / 4$ ), there must be a positive measure of consumers for whom blocking then becomes optimal in equilibrium 26

Additional insights can be obtained if attention competition as in Section 5 is incorporated into the above analysis. In a previous working paper version, we show that if prices are exogenous as in Van Zandt (2004) or Johnson (2013), then mass advertising arises as the unique equilibrium outcome, jointly with a positive measure of blocking consumers. The comparative statics further highlight that a lower salience cost induces an intensified attention competition, which resolves into more consumer blocking and lower firm profits.

\section{Conclusion}

More than a decade ago, a principal analyst at Forrester Research voiced the idea that sending targeted messages could lead to "the end of the era of mass marketing" because "nobody can afford it anymore, and consumers are overloaded with messages". 27 Such a conjecture is indeed supported by standard targeting models that do not account for the possibility of limited attention. With unlimited attention, competitive forces should oblige an advertising firm to target its messages to those consumers it believes it can offer the best deal. In the case of perfect targeting abilities, our model shows that firm targeting matches consumer preferences precisely, resulting in efficient dissemination of information. Anticipating such an equilibrium pattern, consumers become confident that the market will help them identify their most preferred alternative, significantly reducing their propensity to block incoming ads. Overall, the analysis

\footnotetext{
${ }^{26}$ Because consumer expectations about prices could, in principle, be above those ensuring the full coverage of the market $(V-t)$, the uniqueness of the mass advertising equilibrium is not generally assured. Nevertheless, mass advertising is the unique equilibrium if consumers are not arbitrarily pessimistic about high prices (see Proposition A1 in the Appendix).

27 'Cruise ship campaign aims at vacationers tired of snow', The New York Times, Dec 28, 2004.
} 
with unlimited consumer attention depicts targeted advertising as a highly effective marketing instrument, especially given the typical inefficiencies associated with mass advertising (e.g., Grossman and Shapiro, 1984).

Real-world evidence, however, suggests that targeting has evolved quite differently. The advertising industry stands witness to increasing consumer complaints about being overwhelmed by, at best, partially relevant ads, and an increase in ad blocking software. Likewise, "being on the consumers' mind" has remained of deep concern to the advertising firms.

In this article, we have argued that such observations can still be explained within a standard targeting framework, provided limited consumer attention is taken into account. With inattentive consumers and low information costs an efficient, segmenting targeting equilibrium cannot exist because consumers may fail to pay attention to the most useful information, implying that targeted information ceases to work as a reliable shield against business stealing. With limited attention, firms are targeting far less precisely than they could. Moreover, targeting choices are detached from traditional fundamentals, such as preferences or the degree of product differentiation - in an inattentive world, salience is king, despite sophisticated targeting abilities. It follows that the scope for targeting as an efficient and effective marketing instrument may be much smaller, for both firms and consumers, than suggested by previous research. Therefore, it seems vital for empirical work to include reasonable measures to account for consumer inattention when evaluating information and advertising policies.

\section{Appendix}

Proofs of Propositions $\mathbf{1}$ and 2 The claims follow directly from the arguments in the main text; see also the proof of Theorem B1 in the online appendix.

Proof of Theorem 1 We show that Theorem 1 follows from a result in d'Aspremont et al. (1979) and the fact that equilibria in the pricing-targeting game with unlimited attention can be identified using a corresponding complete information game.

Consider a Hotelling setting with two firms $A, B$ at locations $x_{A}, x_{B} \in[0,1], x_{A}<x_{B}$, 
where all consumers $i \in[0,1]$ are ex-ante endowed with complete information about both firms. Both firms simultaneously compete in prices $p_{A}, p_{B}$ with a unit production cost $c>0$. For given prices $\left(p_{j}, p_{-j}\right)$ and $j \in\{A, B\}$, let $\mathcal{O}_{j}\left(p_{j}\right) \equiv\left\{i: U_{i}(j) \geq 0\right\}$ denote the set of consumers who prefer firm $j$ over the outside option, and $\mathcal{P}_{j} \equiv\left\{i: U_{i}(j) \geq U_{i}(-j)\right\}$ is the prime segment of firm $j$, as before. Then, $\lambda\left(\mathcal{P}_{j} \cap \mathcal{O}_{j}\left(p_{j}\right)\right)$ corresponds to firm $j$ 's market demand, and $\tilde{\Pi}_{j}\left(p_{j}, p_{-j}\right)=\left(p_{j}-c\right) \lambda\left(\mathcal{P}_{j} \cap \mathcal{O}_{j}\left(p_{j}\right)\right)$ is firm $j$ 's payoff in the complete information game given prices $\left(p_{j}, p_{-j}\right)$. We say that an equilibrium $\left(p_{A}^{*}, p_{B}^{*}\right)$ of this complete information pricing game is covering if $p_{j}^{*}>c$ and $\lambda\left(\mathcal{O}_{A}\left(p_{A}^{*}\right) \cup \mathcal{O}_{B}\left(p_{B}^{*}\right)\right)=1$. In a covering equilibrium, the set of consumers who do not wish to purchase thus is negligible.

Lemma A1 Suppose that the above complete information pricing game has a unique and covering equilibrium $\left(p_{A}^{*}, p_{B}^{*}\right)$ with $\lambda\left(\mathcal{P}_{j}^{*} \cap \mathcal{O}_{j}\left(p_{j}^{*}\right)\right)>0, j \in\{A, B\}$. Then, the corresponding two-stage pricing-targeting game with unlimited attention has a unique SPE, where first-stage prices are $p_{j}=p_{j}^{*}>c$ and second-stage targeting satisfies $\mathcal{I}_{j}=\mathcal{P}_{j}^{*}, j \in\{A, B\}$.

Proof Consider the pricing-targeting game with ex-ante uninformed but attentive consumers. Given $p_{j}>c$, sequential rationality implies that second-stage targeting satisfies $\lambda\left(\mathcal{I}_{j}\right)=\lambda\left(\mathcal{P}_{j} \cap \mathcal{O}_{j}\left(p_{j}\right)\right)$. This follows because i) it is harmful for firm $j$ to target a positive measure of consumers with $U_{i}(j)<0$, and ii) unlimited attention implies that it is optimal for each firm $j$ to target all consumers with $U_{i}(j) \geq \max \left\{0, U_{i}(-j)\right\}$. Thus, in any subgame following prices $p_{A}, p_{B}>c$, the second-stage payoff of each firm $j$ must be $\Pi_{j}\left(p_{j}, p_{-j}\right)=\left(p_{j}-c\right) \lambda\left(\mathcal{P}_{j} \cap \mathcal{O}_{j}\left(p_{j}\right)\right)$, which formally coincides with the payoff $\tilde{\Pi}_{j}\left(p_{j}, p_{-j}\right)$ from the complete information pricing game. We now show that in the two-stage pricing-targeting game with uninformed consumers, firm $A$ never wants to deviate from the proposed strategy given that $p_{B}=p_{B}^{*}$. Note first that if $A$ plays the proposed equilibrium strategy, then it earns the complete information payoff $\Pi_{j}\left(p_{A}^{*}, p_{B}^{*}\right)=\tilde{\Pi}_{j}\left(p_{A}^{*}, p_{B}^{*}\right)>0$. Any deviation to $p_{A} \leq c$ fails to be profitable, because it cannot lead to a positive payoff. There cannot be a profitable deviation 
with $p_{A}>c$ either, because otherwise

$$
\tilde{\Pi}_{j}\left(p_{A}, p_{B}^{*}\right)=\Pi_{j}\left(p_{A}, p_{B}^{*}\right)>\Pi_{j}\left(p_{A}^{*}, p_{B}^{*}\right)=\tilde{\Pi}_{j}\left(p_{A}^{*}, p_{B}^{*}\right)
$$

which contradicts the presumption that $p_{A}^{*}$ is the unique best-reply to $p_{B}^{*}$ in the complete information pricing game.

As for uniqueness of the SPE, note that there cannot be an SPE where some firm is earning a non-positive profit, as otherwise at least one firm would find it profitable to deviate to some $p=c+\varepsilon$ for sufficiently small $\varepsilon>0$ in the pricing stage, and target accordingly in the second stage. Therefore, in any SPE we must have $p_{A}, p_{B}>c$. Now suppose, by contradiction, that there is an $\mathrm{SPE}$ with $\left(p_{A}, p_{B}\right) \neq\left(p_{A}^{*}, p_{B}^{*}\right)$ in the pricing stage. Then,

$$
\tilde{\Pi}_{j}\left(p_{j}, p_{-j}\right)=\Pi_{j}\left(p_{j}, p_{-j}\right) \geq \max \left\{\Pi_{j}\left(p_{j}^{\prime}, p_{-j}\right), 0\right\} \geq \tilde{\Pi}_{j}\left(p_{j}^{\prime}, p_{-j}\right), \forall p_{j}^{\prime} \geq 0, \forall j \in\{A, B\} .
$$

Hence, $\left(p_{A}, p_{B}\right)$ must also be an equilibrium of the complete information pricing game, contradicting the assumption that this game has $\left(p_{A}^{*}, p_{B}^{*}\right)$ as its unique equilibrium.

In the above Hotelling setting, d'Aspremont et al. (1979) show that if $V>c+t\left(1+\max \left\{x_{B}, 1-\right.\right.$ $\left.x_{A}\right\}$ ) and $\max \left\{x_{A}, 1-x_{B}\right\} \leq 1 / 4$, then the complete information pricing game has a unique equilibrium with prices

$$
p_{A}^{*}=\left(\frac{2}{3}+\frac{x_{A}+x_{B}}{3}\right) t+c, \quad p_{B}^{*}=\left(\frac{4}{3}-\frac{x_{A}+x_{B}}{3}\right) t+c .
$$

Theorem 1 directly follows from Lemma A1 and this result for $x_{A}=0$ and $x_{B}=1$.

Remark Compared to a direct proof, the above also reveals that the segmenting nature of equilibrium targeting in the SPE with unlimited attention does not hinge on the Hotelling setting. Likewise, the coincidence of first-stage equilibrium prices with their counterparts if consumers are endowed with complete information extends beyond Hotelling. The only instance 
where we formally exploited the Hotelling structure in the above proof of Lemma A1 was to establish the intuitive result that there cannot be an SPE with $p_{j} \leq c$ for at least one firm. More precisely, this argument only requires the mild assumption that for any given $p_{-j}$ there is $\varepsilon>0$ such that firm $j$ can assure itself of a positive demand for $p_{j}=c+\varepsilon$. It then follows that whenever the complete information pricing game has a unique and covering Nash equilibrium, the respective pricing-targeting game has a unique SPE, where firms price as in the complete information game, and target their prime segments exclusively in the second stage.

Proof of Theorem 2 Consider a more general setting where firm locations $x_{A}, x_{B} \in[0,1]$, $x_{A}<x_{B}$, are arbitrary. Let $p_{j}^{M}=V-t \cdot \max \left\{x_{j}, 1-x_{j}\right\}$ denote the cutoff price at which all consumers prefer the product of firm $j$ over the outside option. As a simplifying tie-breaking rule, we assume that whenever firms are indifferent about targeting a non-zero measure set of consumers they choose to target that set ${ }^{28}$ We prove the following generalization of Theorem 2. If $c<\pi_{j}\left(p_{j}^{M}-t\right) \forall j \in\{A, B\}$, then the pricing-targeting game with limited attention has a unique SPE, where both firms behave as mass advertisers and charge the monopoly prices, i.e., $\lambda\left(\mathcal{I}_{j}\right)=1$ and $p_{j}^{*}=p_{j}^{M} \forall j \in\{A, B\}$. The statement of Theorem 2 immediately follows by letting $x_{A}=0$ and $x_{B}=1$.

Lemma A2 In any $S P E, \pi_{j} p_{j}>c \forall j \in\{A, B\}$.

Proof As in the proof of Theorem 1, we define $\mathcal{O}_{j}\left(p_{j}\right) \equiv\left\{i: U_{i}(j) \geq 0\right\}$. Consider any pair of prices $\left(p_{j}, p_{-j}\right)$ such that $\pi_{j} p_{j} \leq c$ and $\pi_{-j} p_{-j}>c$. Note that because $\pi_{j} p_{j}^{M}>c$ by presumption, we have $p_{j}<p_{j}^{M}$. With such prices, it is always optimal for firm $-j$ to target the consumers in $\mathcal{O}_{-j}\left(p_{-j}\right)$ exclusively in the second stage, whereas firm $j$ can possibly benefit only from consumers not targeted by $-j$. As a result, the profit of firm $j$ satisfies $\Pi_{j} \leq \max \left\{\left(p_{j}-c\right)\left(1-\lambda\left(\mathcal{O}_{-j}\left(p_{-j}\right)\right)\right), 0\right\}$. Now, consider the deviation where firm $j$ sets the monopoly price $p_{j}^{M}$. Sequential rationality then implies that $j$ targets the entire market in the corresponding subgame (because $c<\pi_{j} p_{j}^{M}$ and $\mathcal{O}_{j}\left(p_{j}^{M}\right)=[0,1]$ ). This yields a deviation payoff $p_{j}^{M}\left(1-\lambda\left(\mathcal{O}_{-j}\left(p_{-j}\right)\right)\right)+\pi_{j} p_{j}^{M} \lambda\left(\mathcal{O}_{-j}\left(p_{-j}\right)\right)-c$, which strictly exceeds $\Pi_{j}$. It follows that any

\footnotetext{
${ }^{28}$ The theorem also holds if we assumed the opposite tie-breaking rule instead, i.e., a firm will choose not to target a consumer if it is indifferent.
} 
price pair $\left(p_{j}, p_{-j}\right)$ with $\pi_{j} p_{j} \leq c$ and $\pi_{-j} p_{-j}>c$ cannot be part of an SPE.

Next, consider any pair of prices $\left(p_{j}, p_{-j}\right)$ such that $\pi_{j} p_{j} \leq c$ and $\pi_{-j} p_{-j}<c$. Because $\pi_{-j} p_{-j}<c$, any equilibrium in the corresponding targeting subgame must be non-overlapping. Thus, with such prices, the profit of firm $j$ must satisfy $\Pi_{j} \leq p_{j}-c$. Consider the deviation of firm $j$ to the price $p_{j}^{M}$. By sequential rationality, $j$ then targets the entire market in the targeting stage, which forces firm $-j$ to shut down. This is a profitable deviation for firm $j$ as $p_{j}^{M}-c>p_{j}-c$. Therefore, there cannot be an SPE where $\pi_{j} p_{j} \leq c$ and $\pi_{-j} p_{-j}<c$.

Finally, consider the pair of prices $\left(p_{A}, p_{B}\right)=\left(\frac{c}{\pi_{A}}, \frac{c}{\pi_{B}}\right)$. Given the tie-breaking assumption that a firm will choose to target a consumer whenever it is indifferent, in the subgame following the above prices both firms will target the entire market and their expected profits are zero. Therefore, firm $A$, for example, can increase its price to $p_{A}^{M}$ and then optimally target the entire market in the second stage. As this is a profitable deviation for firm $A$, there cannot be an SPE where $\pi_{j} p_{j}=c \forall j \in\{A, B\}$.

Lemma A3 In any $S P E, p_{j} \in\left[p_{j}^{M}, V\right] \forall j \in\{A, B\}$.

Proof In any SPE we must have that $p_{j} \leq V$, because otherwise firm $j$ will certainly obtain zero demand. From Lemma A2, we also know that in any SPE, $p_{j}>c / \pi_{j} \forall j \in\{A, B\}$. Then, sequential rationality implies that $\mathcal{I}_{j}=\mathcal{O}_{j}\left(p_{j}\right), \forall j \in\{A, B\}$. Accordingly, for all prices in $\left(c / \pi_{j}, p_{j}^{M}\right]$ firm $j$ optimally chooses to target the entire market, which directly implies that with $p_{j}=p_{j}^{M}$ firm $j$ 's expected profit is strictly higher than with any $p_{j} \in\left(c / \pi_{j}, p_{j}^{M}\right)$. Thus, in any SPE, $p_{j} \in\left[p_{j}^{M}, V\right] \forall j \in\{A, B\}$.

Lemma A4 In any $S P E,\left[0, x_{A}\right] \subset \mathcal{I}_{A}$ and $\left[1-x_{B}, 1\right] \subset \mathcal{I}_{B}$.

Proof According to Lemma A3, in any SPE we must have $p_{j} \geq p_{j}^{M} \forall j \in\{A, B\}$. Further, in any SPE each firm $j \in\{A, B\}$ will target the consumers in $\mathcal{O}_{j}\left(p_{j}\right)$ exclusively in the second stage, where $\mathcal{O}_{j}\left(p_{j}\right)$ is a closed interval around $x_{j}$. Now suppose that $\exists \varepsilon>0$, such that $\lambda\left([0, \varepsilon) \cap \mathcal{I}_{A}\right)=0$ in an SPE. The presumption $c<\pi_{A}\left(p_{A}^{M}-t\right)$ implies that firm $A$ can strictly increase its profit by marginally decreasing its price in the first stage (and target a bit further to its left in the second stage), regardless of whether $\mathcal{O}_{A}\left(p_{A}\right) \subset \mathcal{O}_{B}\left(p_{B}\right)$ or not. This deviation 
incentive directly implies that $\left[0, x_{A}\right] \subset \mathcal{I}_{A}$ in any $\mathrm{SPE} 29$ The proof for $\left[1-x_{B}, 1\right] \subset \mathcal{I}_{B}$ is similar.

Lemma A4 shows that, in any SPE, there must exist $\lambda_{A}, \lambda_{B} \in[0,1]$, such that firm $A$ targets the consumers in $\left[0, \lambda_{A}\right]$, whereas firm $B$ targets the consumers in $\left[1-\lambda_{B}, 1\right]$. Further, it must also hold that $\lambda_{A}+\lambda_{B} \geq 1$. Otherwise, there would be a positive measure set of consumers that are not targeted by either of the two firms. Then, given that $c<\pi_{j}\left(p_{j}^{M}-t\right), \forall j$, and $x_{A}<x_{B}$, firm $A$ (firm $B$ ) has an incentive to lower its price at least marginally, and subsequently target a bit further to its right (left). Now consider any pair of prices such that $p_{j}>p_{j}^{M} \forall j=A, B$. For such prices to be part of an SPE, we must have $\mathcal{I}_{A}=\left[0, \lambda_{A}\right]$ and $\mathcal{I}_{B}=\left[1-\lambda_{B}, 1\right]$, where $\lambda_{A}, \lambda_{B} \in[0,1)$ and $\lambda_{A}+\lambda_{B} \geq 1$ in the subgame following these prices. It is then also necessary that neither firm wants to deviate to a lower price in the first stage. A marginal reduction of the first stage price by firm $j$, together with the corresponding optimal marginal extension of its targeting, implies that, on the margin, $1 / t$ consumers start to prefer firm $j$ over the outside option, yielding a marginal benefit of at least $\pi_{j} p_{j}^{M} / t$. The marginal opportunity costs induced by such a price decrease are $\left(\lambda_{A}+\lambda_{B}-1\right) \pi_{j}+\left(1-\lambda_{-j}\right)+c / t$. Hence, for $\left(p_{A}, p_{B}\right)>\left(p_{A}^{M}, p_{B}^{M}\right)$ to be part of an SPE, we must necessarily have

$$
\pi_{j} p_{j}^{M} / t \leq\left(\lambda_{A}+\lambda_{B}-1\right) \pi_{j}+\left(1-\lambda_{-j}\right)+c / t, \forall j \in\{A, B\}
$$

which further implies that $\pi_{A} p_{A}^{M}+\pi_{B} p_{B}^{M} \leq t+2 c$. However, the assumption that $c<\pi_{j}\left(p_{j}^{M}-t\right)$ $\forall j \in\{A, B\}$ implies $\pi_{A} p_{A}^{M}+\pi_{B} p_{B}^{M}>t+2 c$. We thus reach a contradiction. Hence, there cannot be an SPE where $p_{j}>p_{j}^{M} \forall j \in\{A, B\}$.

In sum, we have shown that in any SPE we must have that $p_{-j}=p_{-j}^{M}$ for at least one firm $-j \in\{A, B\}$. It remains to be shown that it is the unique best reply for firm $j$ to set $p_{j}=p_{j}^{M}$. To see this, first note that if $p_{-j}=p_{-j}^{M}$, then because $c<\pi_{-j} p_{-j}^{M}$ and $\mathcal{O}_{-j}\left(p_{-j}^{M}\right)=[0,1]$ firm $-j$ will optimally choose to target the entire market in the second stage, regardless of firm $j$ 's pricing decision. For firm $j$, any $p_{j} \leq c / \pi_{j}$ will be suboptimal, because (given firm $-j$ 's strategy) its expected profit will be (at most) zero. It is also clear that any $p_{j} \in\left(c / \pi_{j}, p_{j}^{M}\right)$

\footnotetext{
${ }^{29}$ Strictly speaking, the subset relations in Lemma $\mathrm{A} 4$ apply almost surely.
} 
will be strictly dominated by $p_{j}^{M}$, because for all such prices sequential rationality would imply that firm $j$ will also target the entire market in the second stage. Hence, firm $j$ 's best response in the pricing stage can be obtained by solving the problem $\max _{p_{j} \geq p_{j}^{M}} \lambda\left(\mathcal{O}_{j}\left(p_{j}\right)\right)\left(\pi_{j} p_{j}-c\right)$. In other words, firm $j$ 's optimal choice of $p_{j}$ requires it to solve a standard monopoly pricing problem, given its location $x_{j}$ and the fact that any price it chooses will be discounted by $\pi_{j}$. It is then straightforward to verify that, given $c<\pi_{j}\left(p_{j}^{M}-t\right)$, the unique solution to the above maximization problem is $p_{j}=p_{j}^{M}$. Hence, the price pair $\left(p_{A}^{M}, p_{B}^{M}\right)$ is indeed part of an SPE, and it is, actually, the unique SPE outcome.

Remark The analogy to the optimization problem of a complete information monopolist made in the last paragraph of the previous proof holds more generally. To illustrate this, consider an abstract model where consumers are endowed with complete information about a monopoly firm facing a market demand $D_{j}(p)$. Suppose that the monopolist needs to choose the price which maximizes the discounted payoff $\left(\pi_{j} p-c\right) D_{j}(p)$, where $c>0$ is a conventional production cost. If this problem has the full coverage price $p_{j}^{M}$ as its unique solution $\forall j \in$ $\{A, B\}$, then second-stage mass advertising and first-stage monopoly pricing at $p_{j}=p_{j}^{M}$ must be an SPE in the corresponding pricing-targeting game with limited attention and information cost $c$. Applied to the Hotelling model from the main text, it follows that a monopolist in the above complete information setting finds the full coverage price optimal whenever $\pi_{j}(V-2 t)>c$.

Proof of Proposition 3 As there cannot be any positive-measure sets of non-targeted consumers in equilibrium, consider a mutually targeted consumer $i$. Then, (3) is the necessary and sufficient condition for the optimal salience level $s_{j}(i)$ of each firm. There cannot be an asymmetric solution to these equilibrium equations 30 Hence $s_{A}^{*}(i)=s_{B}^{*}(i)=s^{*}$, with $s^{*}>0$ being the unique solution to $p=4 h^{\prime}\left(s^{*}\right) s^{*}$, is the only possible equilibrium salience level. Then, firm $j$ 's expected payoff from a mutually targeted consumer $i$ is

$$
\Pi_{j}(i)=\frac{p}{2}-h\left(s^{*}\right)=2 h^{\prime}\left(s^{*}\right) s^{*}-h\left(s^{*}\right) \geq 2 h\left(s^{*}\right)-h\left(s^{*}\right)=h\left(s^{*}\right)>0,
$$

\footnotetext{
${ }^{30}$ This follows from a result on symmetric contests in Hefti (2017) (Proposition 4), stating that best-reply maps associated with an equation like (3) can never possess asymmetric fixed points.
} 
where the first inequality follows from the convexity of $h(\cdot)$ and $h(0)=0$. Consequently, both firms find it optimal to target the entire market at a uniform salience level $s^{*}$.

Proof of Theorem 3 As in the proof of Theorem 2 consider the general setting where firm locations $x_{A}, x_{B} \in[0,1], x_{A}<x_{B}$, are arbitrary, and thus the cutoff monopoly prices are firmspecific: $p_{j}^{M}=V-t \cdot \max \left\{x_{j}, 1-x_{j}\right\} \forall j=A, B$. We prove the following generalization of Theorem 3. If $\frac{(V-t)^{3}}{(2 V-t)^{2}}>t$, then in any SPE both firms behave as mass advertisers and charge monopoly prices, i.e., $\lambda\left(\mathcal{I}_{j}\right)=1$ and $p_{j}^{*}=p_{j}^{M}, \forall j \in\{A, B\}$, and all consumers are targeted by firm $j \in\{A, B\}$ at the salience level $s_{j}^{*}=\left(\frac{p_{j}^{M}}{p_{j}^{M}+p_{-j}^{M}}\right)^{2} \cdot p_{-j}^{M}$. The statement of Theorem 3 then immediately follows by letting $x_{A}=0$ and $x_{B}=1$.

For given first-stage prices $\left(p_{A}, p_{B}\right) \neq(0,0)$, the salience functions must satisfy condition (3), where the common price $p$ is replaced by the firm-specific price $p_{j}$, and $h^{\prime}(s)=1$. This system of equations has a unique solution given by $s_{j}=p_{-j}\left(\frac{p_{j}}{p_{A}+p_{B}}\right)^{2}>0$. Thus, in the subgame following $\left(p_{A}, p_{B}\right)$, if a consumer is targeted by both firms, then the chosen salience levels of each firm is given by $s_{j}$, independent of that consumer's location. Hence, firm $j$ will capture this consumer's attention with probability $\pi_{j}=\frac{s_{j}}{s_{A}+s_{B}}=\frac{p_{j}}{p_{A}+p_{B}}$. Moreover, if $p_{j}>0$ and $i \in \mathcal{O}_{j}\left(p_{j}\right)$, it is optimal for firm $j$ to target $i$ because even if $i$ is also targeted by firm $-j$

$$
\Pi_{j}(i)=\frac{s_{j}}{s_{A}+s_{B}} \cdot p_{j}-s_{j}=\frac{p_{j}^{2}}{p_{A}+p_{B}}-\frac{p_{j}^{2} \cdot p_{-j}}{\left(p_{A}+p_{B}\right)^{2}}=\frac{p_{j}^{3}}{\left(p_{A}+p_{B}\right)^{2}}>0 .
$$

Hence, sequential rationality implies that in the subgame following the prices $\left(p_{j}, p_{-j}\right)$, the targeting set $\mathcal{I}_{j}$ for firm $j$ can only differ from $\mathcal{O}_{j}\left(p_{j}\right)$ up to a zero-measure set. We now claim that, regardless of the price $p_{-j}$ chosen by firm $-j$, firm $j$ 's best response in the first stage must satisfy $p_{j} \in\left[p_{j}^{M}, V\right]$. It is clear that no price $p_{j} \in\{0\} \cup(V,+\infty)$ can be a best response for firm $j$, because with such a price firm $j$ will for sure earn zero profit (whereas by choosing $p_{j}=p_{j}^{M}$ it can secure a strictly positive profit). Next, consider any price $p_{j} \in\left(0, p_{j}^{M}\right)$. With such a price, all informed consumers will prefer firm $j$ over the outside option, and it would be indeed for firm $j$ to target the entire market. The expected total profit of firm $j$, given 
sequential rationality of firm $-j$ 's strategy, then is given by

$$
\Pi_{j}=\frac{p_{j}^{3}}{\left(p_{A}+p_{B}\right)^{2}} \cdot \lambda\left(\mathcal{O}_{-j}\left(p_{-j}\right)\right)+p_{j} \cdot\left(1-\lambda\left(\mathcal{O}_{-j}\left(p_{-j}\right)\right)\right) .
$$

It is straightforward to show that the above function $\Pi_{j}$ is strictly increasing in $p_{j}$. Therefore, we can assume that each firm $j$ is constrained to choosing a price $p_{j} \in\left[p_{j}^{M}, V\right]$ in the first stage. Now take $p_{-j} \in\left[p_{-j}^{M}, V\right]$ as given and consider firm $j$ 's pricing strategy. Starting from some price $p_{j} \in\left(p_{j}^{M}, V\right]$ and applying the envelope theorem, we can assert that by marginally lowering its price and then targeting a bit further either to its left or right, the additional revenue that firm $j$ can obtain is at least $\frac{s_{j}}{s_{j}+s_{-j}} \cdot p_{j} \cdot \frac{1}{t}=\frac{p_{j}^{2}}{\left(p_{j}+p_{-j}\right) t}$. Meanwhile, the marginal opportunity cost of doing so is at most $1+\frac{h\left(s_{j}\right)}{t}=1+\frac{p_{j}^{2} p_{-j}}{\left(p_{j}+p_{-j}\right)^{2} t}$. Hence, for firm $j$ not to have the incentive to lower its price, it is necessary that

$$
\frac{p_{j}^{2}}{p_{j}+p_{-j}} \leq t+\frac{p_{j}^{2} p_{-j}}{\left(p_{j}+p_{-j}\right)^{2}} \Longleftrightarrow \frac{p_{j}^{3}}{\left(p_{j}+p_{-j}\right)^{2}} \leq t
$$

Because $p_{j}>p_{j}^{M} \geq V-t$ and $p_{-j} \leq V$, condition A.2 will be violated for sure if $\frac{(V-t)^{3}}{(2 V-t)^{2}}>t$, or, equivalently, $\frac{(V / t-1)^{3}}{(2 V / t-1)^{2}}>1$. Hence, whenever the last inequality holds firm $j$ 's unique best response in the first stage is to choose $p_{j}=p_{j}^{M}$ for all $p_{-j} \in\left[p_{-j}^{M}, V\right]$. It is a standard numeric exercise to show that $(x-1)^{3}=(2 x-1)^{2}$ has a unique real-valued solution approximately given by $x=5.86$, and therefore $(x-1)^{3}>(2 x-1)^{2}$ whenever $x \geq 5.9$. The parametric requirement $V / t \geq 5.9$ now follows by setting $x=V / t$.

Proof of Theorem 4 Suppose that an equilibrium with prices $\left(p_{A}^{*}, p_{B}^{*}\right)$ exists, where a positive measure set of consumers $\tilde{P}_{j} \subset \mathcal{P}_{j}^{*}$ are targeted by firm $-j$. This is rational for firm $-j$ only if (i) these consumers are not targeted by firm $j$, (ii) the probability that these consumers will not use the ad blocker, $\alpha^{*} \in(0,1]$, is sufficiently high. Sequential rationality and $\lambda\left(\tilde{P}_{j}\right)>0$ then imply that we must have $p_{j}^{*} \leq c / \alpha^{*} \leq p_{-j}^{*}<V$. Hence, in such an equilibrium, the expected profit of firm $j$ must be zero. By deviating to the price $p_{j}=p_{-j}^{*}+\varepsilon$ for a sufficiently small $\varepsilon>0$ and by subsequently targeting consumers who are sufficiently close to $x_{j}$, firm $j$ can 
secure itself a strictly positive profit. Therefore, there cannot be an equilibrium with intrusive targeting.

Now suppose that consumers expect the equilibrium prices to be $t+c$, and that each firm targets its closest half of the market. With this expectation, the expected (ex-ante) utility from listening for a given $\gamma_{i}$ is

$$
E U\left(b_{i}=0 \mid \gamma_{i}\right)=V-(t+c)-\mathbb{E}[\min \{i, 1-i\}] \cdot t-\gamma_{i}=V-\frac{5 t}{4}-c-\gamma_{i},
$$

where $\mathbb{E}[\min \{i, 1-i\}] t=1 / 4 t$ is the expected disutility of each consumer from consuming at her first-best firm (as each consumer is ex-ante uninformed about her location on the Hotelling line relative to the firms). It follows that no consumer blocks whenever $V-5 t / 4-c \geq \bar{\gamma}$. From the firm-side viewpoint, the game is then identical to the one without the blocking option, meaning that the proposed firm strategies must be mutual best replies by Theorem 1. As consumer expectations are correct, these strategies together with $b_{i}=0 \forall i$ constitute an equilibrium of the pricing-targeting-blocking game.

Proof of Theorem 5 First, suppose that consumers expect to receive information from both firms, and that both firms charge the monopoly price $p^{M}=V-t$. Then

$$
E U_{i}\left(b_{i}=0 \mid \gamma_{i}\right)=V-(V-t)-\mathbb{E}\left[\frac{i}{2}+\frac{1-i}{2}\right] t-2 \gamma_{i}=t / 2-2 \gamma_{i}
$$

Hence, when expecting the firms to play the above strategies, a consumer does not block if and only if $\gamma_{i} \leq t / 4 \equiv \hat{\gamma}$. Next, suppose that a fraction $1-F(\hat{\gamma})$ of consumers block, and firm $-j$ chooses $p_{-j}=p^{M}$ in the pricing stage. As $c<\frac{F(\hat{\gamma})}{2} p^{M}$ by presumption, sequential rationality implies that firm $-j$ will target the entire market, regardless of the price chosen by firm $j$. But then, the strategic problem of firm $j$ is similar to the one in the game with price competition from Section 4, assuming that its perception probability is exogenously set to $\pi_{j}=\frac{F(\hat{\gamma})}{2}$ in that game. Hence, from the proof of Theorem 2 , we can conclude that based on the assumption $c<\frac{F(\hat{\gamma})}{2}\left(p^{M}-t\right)$, it is the unique best reply for firm $j$ to choose $p_{j}=p^{M}$ in the 
pricing stage, and to advertise to the entire market in the targeting stage (and vice-versa for firm $-j$ ). As consumer expectations are correct, the proposed strategy profile must constitute an equilibrium.

Further, whenever $F(\hat{\gamma})<1$, a positive measure set of consumers block in the above mass advertising equilibrium. Suppose now that there also is an equilibrium without blocking. Given that no consumer blocks, the condition $c<\frac{F(\hat{\gamma})}{2}\left(p^{M}-t\right)<\frac{1}{2}\left(p^{M}-t\right)$ implies, by Theorem 2 , that the only equilibrium candidate strategies for the firms are that both choose the monopoly price $p^{M}$ in the first stage, and mass advertise in the second stage. If consumers correctly anticipate this behavior, it follows that a fraction $1-F(\hat{\gamma})>0$ of consumers would find blocking optimal, which contradicts the supposed existence of an equilibrium without any blocking.

Remark on Equilibrium Uniqueness Let $p^{E}$ be the price that consumers expect to pay in the market. The following result shows that whenever the cost condition of Theorem5holds and consumers are not too pessimistic about high prices, the mass advertising equilibrium identified by Theorem 5 must be the unique equilibrium in the pricing-targeting-blocking game.

Proposition A1 If $p^{E} \leq V-t$, then the equilibrium in Theorem 5 is the unique equilibrium of the pricing-targeting-blocking game with limited attention.

Proof If $p^{E} \leq V-t$, then a fraction $\alpha \geq F(\hat{\gamma})$ of consumers choose to listen. Thus, a firm's effective perception chance from targeting a consumer $i$ is at least $\hat{\pi}_{j}=\alpha \pi_{j}=\alpha / 2\left(\hat{\pi}_{j}=\alpha\right.$ if $\left.g_{-j}(i)=0\right)$. Then, Theorem 2 proves that mass advertising at the monopoly price is the unique equilibrium outcome whenever $c<\hat{\pi}_{j}(V-2 t)$. However, this inequality is implied by the presumption of Theorem 5 and the fact that $\alpha \geq F(\hat{\gamma})$. Thus (second-stage) mass advertising and (first-stage) monopoly pricing constitute the unique equilibrium outcome of the game. In turn, the only price expectation consistent with this behavior has $p^{E}=p^{M}=V-t$. 


\section{References}

Anderson, S. P. and A. De Palma (2009). Information congestion. The RAND Journal of Economics 40, 688-709.

Anderson, S. P. and A. De Palma (2012). Competition for attention in the information (overload) age. The RAND Journal of Economics 43, 1-25.

Anderson, S. P. and J. S. Gans (2011). Platform siphoning: ad-avoidance and media content. American Economic Journal: Microeconomics 3, 1-34.

Armstrong, M., J. Vickers, and J. Zhou (2009). Consumer protection and the incentive to become informed. Journal of the European Economic Association 7, 399-410.

Athey, S. and J. S. Gans (2010). The impact of targeting technology on advertising markets and media competition. American Economic Review (Papers and Proceedings) 100, 608.

Bergemann, D. and A. Bonatti (2011). Targeting in advertising markets: implications for offline versus online media. The RAND Journal of Economics 42, 417-443.

Brahim, N. B. E.-B., R. Lahmandi-Ayed, and D. Laussel (2011). Is targeted advertising always beneficial? International Journal of Industrial Organization 29, 678-689.

d'Aspremont, C., J. J. Gabszewicz, and J.-F. Thisse (1979). On hotelling's "stability in competition". Econometrica 47, 1145-1150.

Ellison, G. (2006). Bounded rationality in industrial organization. In R. Blundell, W. Newey, and T. Persson (Eds.), Advances in Economics and Econometrics: Theory and Applications, Ninth World Congress, Volume 2. Cambridge University Press.

Esteban, L., A. Gil, and J. M. Hernandez (2001). Informative advertising and optimal targeting in a monopoly. Journal of Industrial Economics 49, 161-180.

Esteban, L. and J. M. Hernandez (2007). Strategic targeted advertising and market fragmentation. Economics Bulletin 12, 1-12. 
Esteves, R.-B. and J. Resende (2016). Competitive targeted advertising with price discrimination. Marketing Science 4, 576-587.

Evans, D. S. (2009). The online advertising industry: economics, evolution, and privacy. Journal of Economic Perspectives 23, 37-60.

Falkinger, J. (2007). Attention economies. Journal of Economic Theory 133, 266-294.

Farahat, A. (2013). How effective is targeted advertising? In American Control Conference (ACC), 2013, pp. 6014-6021. IEEE.

Ghose, A. M. and S. Yang (2009). An empirical analysis of search engine advertising: sponsored search in electronic markets. Management Science 55, 1605-1622.

Golosov, M. and J. Lucas, Robert E. (2007). Menu costs and phillips curves. Journal of Political Economy 115, 171-199.

Gritckevich, A., Z. Katona, and M. Sarvary (2018). Ad blocking. Mimeo.

Grossman, G. M. and C. Shapiro (1984). Informative advertising with differentiated products. Review of Economic Studies 51, 63-81.

Gu, B., P. Konana, and H.-W. M. Chen (2012). Identifying consumer consideration set at the purchase time from aggregate purchase data in online retailing. Decision Support Systems 53, 625-633.

Hefti, A. (2017). Equilibria in symmetric games: theory and applications. Theoretical Economics 12, 979-1002.

Hefti, A. (2018). Limited attention, competition and welfare. Journal of Economic Theory 178, 318-359.

Hefti, A. and S. Heinke (2015). On the economics of superabundant information and scarce attention. Oeconomia 5, 37-74. 
Heidhues, P. and B. Köszegi (2018). Behavioral industrial organization. In D. Bernheim, S. Della-Vigna, and D. Laibson (Eds.), Hanbook of Behavioral Economics, Volume 1, Chapter 6. Elsevier.

Hu, Y., J. Shin, and Z. Tang (2015). Incentive problems in performance-based online advertising pricing: cost per click vs. cost per action. Management Science 62, 2022-2038.

Iyer, G., D. Soberman, and J. M. Villas-Boas (2005). The targeting of advertising. Marketing Science 24, 461-476.

Johnson, J. P. (2013). Targeted advertising and advertising avoidance. The RAND Journal of Economics 44, 128-144.

Miller, G. A. (1956). The magical number seven, plus or minus two: some limits on our capacity for processing information. Psychological Review 63, 81-97.

Roy, S. (2000). Strategic segmentation of a market. International Journal of Industrial Organization 18, 1279-1290.

Sims, C. A. (2003). The implications of rational inattention. Journal of Monetary Economics 50, $665-690$.

Spiegler, R. (2011). Bounded Rationality and Industrial Organization. OUP USA.

Van Zandt, T. (2004). Information overload in a network of targeted communication. The RAND Journal of Economics 35, 542-560.

Yao, S. and C. F. Mela (2011). A dynamic model of sponsored search advertising. Marketing Science 30, 447-468. 


\title{
Online Appendix - Not for Publication \\ Targeted Information and Limited Attention
}

\author{
Andreas Hefti and Shuo Liu
}

In this Appendix we provide the following supplementary material: i) a characterization of all targeting equilibria that arise by abstracting from price competition, ii) an extension to imperfect marketing data, iii) an analysis of the pricing-targeting game with a different time structure than in the main text, and iv) a discussion of persuasive advertising in the context of our model.

\section{B.1 Targeting Equilibrium: A Characterization}

In this section, we extend our baseline targeting model from Section 3 of the main text with a number of features. First, we introduce consumer heterogeneity in attention by supposing that the attention capacity $R_{i} \in\{1,2\}$ is independently distributed over the population, where the probability that $R_{i}=1$ is $q \in[0,1]$. Second, we allow for heterogeneity in information costs $c_{j}>0$, and arbitrary firm locations $x_{A}, x_{B} \in[0,1], x_{A}<x_{B}$. Prime segments are separated by the indifferent consumer (1) where, for simplicity, we suppose that each firm earns a fixed value $p_{j}>c_{j}$ for any successful transaction. More generally, the next theorem applies whenever a consumer population can be decomposed in prime segments, such that $\lambda\left(\mathcal{P}_{1} \cap \mathcal{P}_{2}\right)=0$ and 
$\mathcal{P}_{1} \cup \mathcal{P}_{2}=[0,1] !^{1}$ Under the above assumptions, we can provide a full characterization of the equilibrium targeting profiles given information costs, consumer inattention $q$, and perception probabilities.

Theorem B1 (Targeting Equilibria) Let $p_{j}>c_{j}>0$ and $\pi_{j} \in(0,1)$ for $j=A, B$.

(i) Segmenting If $c_{j}>q \pi_{j} p_{j}$ and $c_{j}<q \pi_{j} p_{j}+(1-q) p_{j} \forall j \in\{A, B\}$, then a unique and non-intrusive targeting equilibrium exists, where $\lambda\left(\mathcal{I}_{j} \cap \mathcal{P}_{-j}\right)=0$, and $\lambda\left(\mathcal{I}_{j} \cap \mathcal{P}_{j}\right)=\lambda\left(\mathcal{P}_{j}\right)$, $\forall j \in\{A, B\}$

(ii) Total Mass Advertising If $c_{j}<q \pi_{j} p_{j} \forall j \in\{A, B\}$, then both firms behave like mass advertisers in the unique targeting equilibrium, i.e., $\lambda\left(\mathcal{I}_{j}\right)=1 \forall j \in\{A, B\}$.

(iii) Coordination Let $c_{j}>q \pi_{j} p_{j}$ and $c_{-j}>q \pi_{-j} p_{-j}+(1-q) p_{-j}$. If $c_{j}<q \pi_{j} p_{j}+(1-q) p_{j}$, then any non-overlapping targeting profile satisfying $\lambda\left(\mathcal{I}_{j} \cap \mathcal{P}_{j}\right)=\lambda\left(\mathcal{P}_{j}\right)$ and $\lambda\left(\mathcal{I}_{A} \cup \mathcal{I}_{B}\right)=1$ constitutes an equilibrium. If instead $c_{j}>q \pi_{j} p_{j}+(1-q) p_{j}$, then any non-overlapping targeting profile that satisfies $\lambda\left(\mathcal{I}_{A} \cup \mathcal{I}_{B}\right)=1$ constitutes an equilibrium.

(iv) Partial Mass Advertising If $c_{j}<q \pi_{j} p_{j}$ and $c_{-j} \in\left(q \pi_{-j} p_{-j}, q \pi_{-j} p_{-j}+(1-q) p_{-j}\right)$, then a unique targeting equilibrium exists, and $\lambda\left(\mathcal{I}_{j}\right)=1, \lambda\left(\mathcal{I}_{-j} \cap \mathcal{P}_{-j}\right)=\lambda\left(\mathcal{P}_{-j}\right), \lambda\left(\mathcal{I}_{-j} \cap \mathcal{P}_{j}\right)=$ 0. If instead $c_{j}<q \pi_{j} p_{j}$ and $c_{-j}>q \pi_{-j} p_{-j}+(1-q) p_{-j}$, then again a unique targeting equilibrium exists, and $\lambda\left(\mathcal{I}_{j}\right)=1, \lambda\left(\mathcal{I}_{-j}\right)=0$.

Proof To see (i), note that if $c_{j}<q \pi_{j} p_{j}+(1-q) p_{j}$ it is never optimal for firm $j$ to leave a positive measure set of consumers in its prime segment uninformed; by targeting these consumers, firm $j$ obtains a strictly positive expected payoff. But if each firm covers its respective prime segment, intrusive targeting necessarily yields a strictly negative payoff whenever $c_{j}>q \pi_{j} p_{j}$.

\footnotetext{
${ }^{1}$ For example, prime segments could correspond to voter preferences, and politicians need to decide which voters to target.
} 
Thus (i) follows. Next, if $c_{j}<q \pi_{j} p_{j}$, then targeting the entire market $\left(\lambda\left(\mathcal{I}_{j}\right)=1\right)$ is a strictly dominant strategy for firm $j$, as $j$ can always secure itself a positive expected payoff for any consumer it targets. Thus (ii) follows.

We now prove (iii). If $c_{-j}>q \pi_{-j} p_{-j}+(1-q) p_{-j}$, targeting any positive measure set of consumers is profitable for firm $-j$ iff these are not targeted by firm $j$. If $c_{j}>q \pi_{j} p_{j}$ and $c_{j}<q \pi_{j} p_{j}+(1-q) p_{j}$, then it is never be optimal for firm $j$ to leave a positive measure set of consumers in $\mathcal{P}_{j}$ uninformed about its product by the latter inequality. In addition, the condition $c_{j}>q \pi_{j} p_{j}$ implies that targeting any positive measure set of consumers in $\mathcal{P}_{-j}$ is profitable for $j$ iff these are not targeted by firm $-j$. The first part of (iii) thus immediately follows. For the second part, note that if instead $c_{j}>q \pi_{j} p_{j}+(1-q) p_{j}$, then the strategic consideration of firm $j$ is identical to that of firm $-j$. Thus, a targeting profile is an equilibrium iff it is non-overlapping and does not leave any positive measure set of consumers untargeted.

Finally, for (iv), note that if $c_{j}<q \pi_{j} p_{j}$, then targeting the entire market is a dominant strategy for firm $j$. Then, targeting consumers in $\mathcal{P}_{j}$ is harmful for firm $-j$ whenever $c_{-j}>$ $q \pi_{-j} p_{-j}$. Hence firm $-j$ will never intrude. If, in addition, $c_{-j}>q \pi_{-j} p_{j}+(1-q) p_{-j}$, then targeting consumers in $\mathcal{P}_{-j}$ is harmful for firm $-j$ given firm $j$ 's dominant strategy of targeting the entire market. Hence, firm $-j$ is forced to shut down. If instead $c_{-j} \in\left(q \pi_{-j} p_{-j}, q \pi_{-j} p_{-j}+\right.$ $\left.(1-q) p_{-j}\right)$, then targeting $\mathcal{P}_{-j}$ exclusively is the unique best reply of firm $-j$.

Discussion Theorem B1 shows that consumer inattention has a radical effect on the nature of the targeting equilibrium. In particular, the segmenting equilibrium (i) is only assured to occur in the extreme case where $q=0$. Whenever $q>0$, there always is a range of positive information costs, such that an inattention-specific equilibrium arises. This is illustrated in Figure 1 (a) in the symmetric case where $c_{j}=c, p_{j}=p$ and $\pi_{j}=1 / 2, j=A, B$. Note that even if $q>0$ is small, the mass advertising equilibrium (ii) is the unique equilibrium prediction 


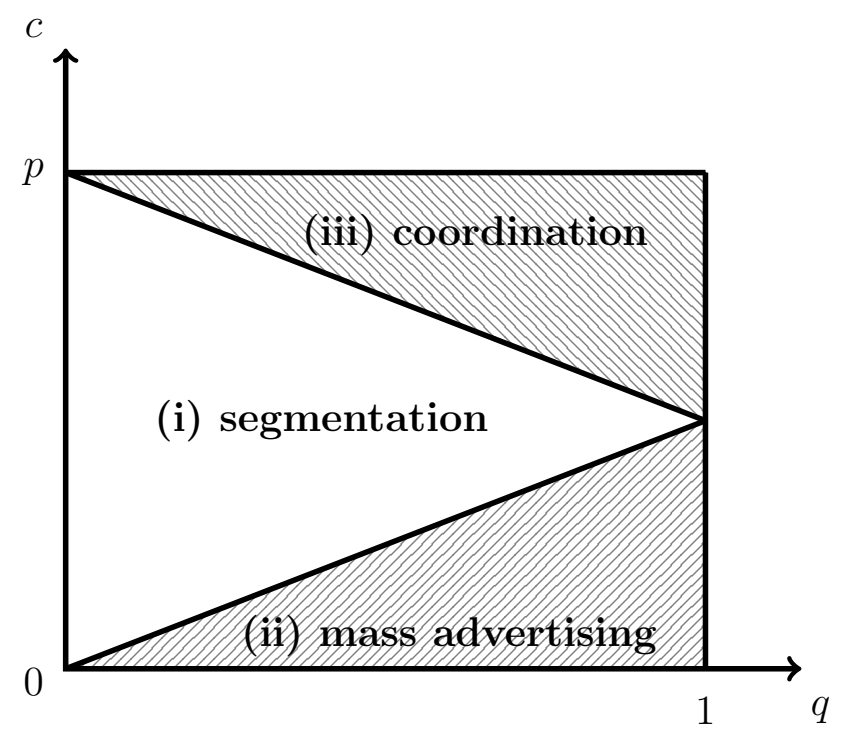

(a) $c_{j}=c, p_{j}=p, \pi_{j}=\frac{1}{2} \forall j=A, B$.

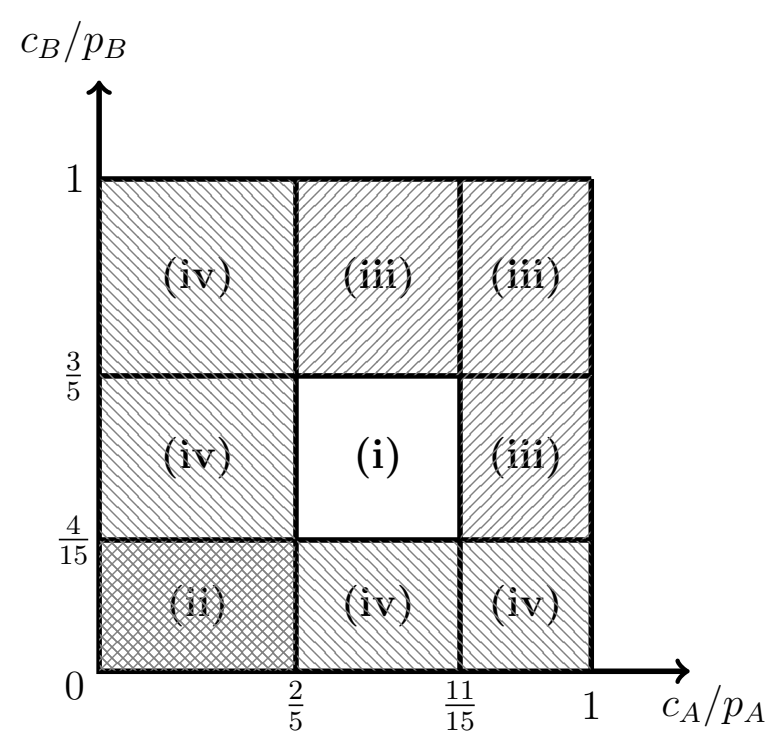

(b) $\pi_{A}=\frac{3}{5}, \pi_{B}=\frac{2}{5}$, and $q=\frac{2}{3}$.

Figure 1: Equilibrium Regimes.

for low enough information costs. Figure 1(b) illustrates the equilibrium regions given that $\pi_{A}=3 / 5, \pi_{B}=2 / 5$ and $q=2 / 3$. With sufficiently low information costs, so that (iii) does not apply, any equilibrium necessarily features intrusive targeting because at least one firm mass advertises. By contrast, with $q>0$ and sufficiently high information costs, the non-overlapping coordination equilibria (iii) arise, because the information expenditures for mutually targeted consumers are not covered anymore. Although a theoretical possibility, we emphasize that the coordination equilibria (iii) are not robust. Specifically, they cannot arise with an endogenous information cost due to attention competition (as in Section 5), or if firms decide sequentially on their targeting strategies, and they also vanish with the pay-per-click cost structure, where marginal information costs are $c_{j}\left(\pi_{j} q+(1-q)\right)$ (instead of $\left.c_{j}\right)$.

Further, Theorem B1 suggests that comparative advantages in attention-seeking become increasingly relevant for a firm's market share, as opposed to prime segments. For example, in the mass advertising equilibrium (ii), each firm's market share is $m_{j}=q \pi_{j}+(1-q) \lambda\left(\mathcal{P}_{j}\right)$, which is increasing in $\pi_{j}$ if $q>0$. Likewise, $m_{j}$ increases in inattention $q$ if and only if $\pi_{j} \geq \lambda\left(\mathcal{P}_{j}\right)$. 
Hence, unless $\pi_{j}=\lambda\left(\mathcal{P}_{j}\right) \forall j \in\{A, B\}$, an increase in $q$ implies a favorable redistribution of market shares and profits to the firm that has a comparative advantage in attention-seeking. Finally, the presence of inattentive consumers can indirectly harm the attentive ones by alluring firms to fill the mailbox of every consumer. In particular, a small increase in $q$ may discontinuously alter the equilibrium from segmenting to mass advertising, which is undesirable for all inattentive consumers and, in presence of a nuisance cost of advertising, also would impose a search externality on attentive consumers (Armstrong, 2015).

\section{B.2 Imperfect Marketing Data}

In the following, we consider how limited attention affects the targeting incentives if the firms are not endowed with perfect targeting abilities. In reality, firms need to acquire consumer marketing data, which raises the additional question if consumer inattention affects the firmside information gathering process ${ }^{2}$ The main insights of this section are i) the effect of limited attention on the targeting behavior is essentially the same as in the baseline model, and ii) the incentives to acquire more precise marketing data depends on consumer attention.

We again consider two firms $A$ and $B$, and a continuum of consumers indexed by $i \in[0,1]$. For simplicity, we assume that each firm $j$ earns an exogenous revenue $p_{j}>c$ from any successful transaction, where $c>0$ again is the marginal information cost. Consistent with the baseline model, each consumer has a strict preference $\succ_{i}$ defined over two firms $A$ and $B$, where $A \succ_{i} B$ if $i \in \mathcal{P}_{A}=[0,1 / 2]$ and $B \succ_{i} A$ if $i \in \mathcal{P}_{B}=(1 / 2,1]$. Other than in the baseline model, each firm now may possess imperfect knowledge about consumer preferences, capturing a limitation in the precision of the marketing data. We assume that the marketing data of each firm always

\footnotetext{
${ }^{2}$ In most cases, firms collect consumer data either from a direct firm-customer relationship ("first-party" data) or by acquiring data from specialized companies ("third-party" data). Third-party data usually is collected on the Internet using digital cookies, web beacons or e-tags without consumers being aware of them (see 'Advertising and technology', The Economist, Sep 2014, Special Report).
} 
comprises all relevant consumers in thus that consumers with zero valuation for a product are always excluded. As targeting is costly, this assumption is not critical for our purpose $3^{3}$ We suppose that the marketing data takes on the form of a binary signal $z_{j i} \in\{0,1\}$ for each consumer with the following structure: If $j \succ_{i}-j$, then $z_{j i}=1$ with probability 1 ; if $-j \succ_{i} j$, then $z_{j i}=1$ with probability $\alpha_{j} \in[0,1]$. The marketing data thus qualitatively follows the true preference distribution, but may exaggerate the preferences for some consumers. If $z_{j i}=0$, then $j$ infers that $i \in \mathcal{P}_{-j}$, and accordingly $z_{-j i}=1$. Thus, $\alpha_{j}$ yields a simple measure of $j$ 's data quality, where a smaller $\alpha_{j}$ means that firm $j$ is more likely to correctly separate non-prime from prime consumers. For $\alpha_{j}=0$ the model collapses to the baseline case, whereas firm $j$ has no clue about consumer tastes if $\alpha_{j}=1$.

Given its marketing data, each firm independently decides which consumers to target, and the targeting profile $\left(g_{A}, g_{B}\right)$ determines the consumers' information sets. Let $\mathcal{P}_{j}^{z}=\{i \in$ $\left.[0,1]: z_{j i}=1\right\}$ denote $j$ 's prime segment as indicated by its data. Note that $\mathcal{P}_{j} \subseteq \mathcal{P}_{j}^{z}$ and $\lambda\left(\mathcal{P}_{A}^{z} \cup \mathcal{P}_{B}^{z}\right)=1$. Given the general understanding that modern media has lowered information costs, we restrict the following analysis to the case of cheap information provision. We first show how the marketing data affects the targeting equilibrium with attentive consumers.

Proposition B1 Suppose that $c<p_{j} /\left(1+\alpha_{j}\right) \forall j \in\{A, B\}$. The targeting game with imperfect marketing data and unlimited attention has a unique equilibrium, and each firm only targets its indicated prime segment, i.e. $\lambda\left(\mathcal{I}_{j} \cap \mathcal{P}_{j}^{z}\right)=\lambda\left(\mathcal{P}_{j}^{z}\right)$ and $\lambda\left(\mathcal{I}_{j} \backslash \mathcal{P}_{j}^{z}\right)=0$. The equilibrium profits are $\Pi_{j}=p_{j} \lambda\left(\mathcal{P}_{j}\right)-c \lambda\left(\mathcal{P}_{j}^{z}\right), \forall j \in\{A, B\}$.

Proof Given a signal $z_{j i}=1$, the probability that $j \succ_{i}-j$ is given by $1 /\left(1+\alpha_{j}\right) \geq 1 / 2$ according to Bayes' rule. Firm $j$ 's expected payoff from targeting such a consumer is at least

\footnotetext{
${ }^{3} \mathrm{~A}$ firm is always harmed by targeting a consumer with zero interest, independent of consumer attention, meaning that data revealing such consumers is always valuable.
} 
$p_{j} /\left(1+\alpha_{j}\right)-c$, and hence strictly positive given that $p_{j}>\left(1+\alpha_{j}\right) c$. Thus, firm $j$ rationally targets any consumer for which $z_{j i}=1$. Now suppose $z_{j i}=0$. In this case, firm $j$ can conclude that $-j \succ_{i} j$, which further implies that, given the structure of the data, firm $j$ also knows that $z_{-j i}=1$ with probability one. Thus, $j$ correctly anticipates that this consumer will be targeted by firm $-j$ by the previous argument. Given the assumption of unlimited attention, firm $j$ therefore abstains from targeting such consumers. Hence $z_{j i}=0 \Longrightarrow g_{j}(i)=0$ and $z_{j i}=1 \Longrightarrow g_{j}(i)=1$ for almost every $i \in[0,1]$.

Proposition B1 shows that, different to the baseline model, the equilibrium targeting profile is overlapping despite fully attentive consumers. The crucial difference to the overlapping targeting profile under limited attention identified earlier is that the overlap in Proposition B1 is entirely driven by imprecise information about the market and not by a strategic desire to invade the competitor's prime segment. Thus, the baseline result about equilibrium targeting with attentive consumers continues to hold in thus that no firm seeks to intrude the prime segment of the other firm to the best of its knowledge. Therefore, any (unilateral) improvement in the marketing data, as measured by a lower $\alpha_{j}$, reduces overlapping and wasteful targeting, and increases the firm's profit. Each firm thus has an incentive to acquire better data.

We now reconsider the above targeting decisions with limited attention. The following result shows that mass advertising remains the unique equilibrium prediction by the same low cost condition identified in Proposition 2, independent of the data quality.

Proposition B2 Suppose that $c<\pi_{j} p_{j} \forall j \in\{A, B\}$. The targeting game with imperfect marketing data and limited attention has a unique equilibrium, and each firm advertises to the entire market, i.e., $\lambda\left(\mathcal{I}_{j}\right)=1$. The equilibrium profits are $\Pi_{j}=p_{j} \lambda\left(\mathcal{P}_{j}\right)-c, \forall j \in\{A, B\}$.

Proof Analogous to the proof of Proposition 2. 
The reason for the mass advertising equilibrium is that the firms' targeting decisions are guided by attentional concerns rather than by preferences. It follows that more precise marketing data fails to reduce the targeting overlap, in contrast to the case of unlimited attention. Moreover, limited consumer attention implies that the firms have no incentives to acquire marketing data which more precisely separates prime from non-prime consumers.

\section{B.3 Simultaneous Pricing and Targeting}

Consider the pricing-targeting game studied in Section 2, but with the modification that firms choose their prices $p_{j} \in \mathbb{R}_{+}$and targeting functions $g_{j}:[0,1] \rightarrow\{0,1\}$ simultaneously. Let firm locations be $x_{A}, x_{B} \in[0,1], x_{A}<x_{B}$, and $p_{j}^{M} \equiv V-t\left(1-\max \left\{x_{j}, 1-x_{j}\right\}\right), \forall j \in\{A, B\}$, as before. We show that, similar to the two-stage game, both firms behave like mass advertisers in the unique equilibrium of the simultaneous pricing-targeting game with limited attention.

Proposition B3 If $c<\pi_{j}\left(p_{j}^{M}-t\right) \forall j \in\{A, B\}$, then there is a unique Nash equilibrium in the simultaneous pricing-targeting game with limited attention, where both firms behave as mass advertisers, and charge the monopoly prices, i.e., $\lambda\left(\mathcal{I}_{j}\right)=1$ and $p_{j}^{*}=p_{j}^{M}, \forall j \in\{A, B\}$.

Proof Existence of the claimed equilibrium: If $p_{-j}=p_{-j}^{M}$ and firm $-j$ is targeting the entire market then, given that $c<\pi_{j}\left(p_{j}^{M}-t\right)$, it is the unique best reply of firm $j$ to choose $p_{j}=p_{j}^{M}$ and target the entire market as well. Hence, the proposed strategy profile constitutes a Nash equilibrium in the simultaneous game. For uniqueness, take firm $-j$ 's strategy $\left(p_{-j}, g_{-j}\right)$ as given. Then, only prices $p_{j} \in \mathbb{R}_{+}$with $p_{j} \geq p_{j}^{M}$ can be part of firm $j$ 's best reply. To see this, note that by choosing $p_{j}=p_{j}^{M}$ and targeting the entire market, firm $j$ can secure an expected profit of $\hat{\Pi}_{j}=p_{j}^{M}\left(\pi_{j} \lambda\left(\mathcal{I}_{-j}\right)+1-\lambda\left(\mathcal{I}_{-j}\right)\right)-c>0$. Therefore, no price $p_{j}<c$ can be part of firm 
$j$ 's best reply. Further, it is optimal for $j$ to complement any price $p_{j} \in\left(c / \pi_{j}, p_{j}^{M}\right)$ with targeting the entire market. This yields an expected profit $\Pi_{j}^{D}\left(p_{j}\right)=p_{j}\left(\pi_{j} \lambda\left(\mathcal{I}_{-j}\right)+1-\lambda\left(\mathcal{I}_{-j}\right)\right)-c$, which is strictly less than $\hat{\Pi}_{j}$, showing that any $p_{j} \in\left(c / \pi_{j}, p_{j}^{M}\right)$ is less desirable than $p_{j}^{M}$. Any price $p_{j} \in\left[c, c / \pi_{j}\right]$ is dominated by $p_{j}^{M}$ as well, because the highest expected profit such a price can lead to is $\Pi_{j}^{D}\left(p_{j}\right)=\left(1-\lambda\left(\mathcal{I}_{-j}\right)\right)\left(p_{j}-c\right)$ (i.e., when firm $j$ targets all the consumers that are not targeted by $-j$ ), which is strictly less than $\hat{\Pi}_{j}$. We conclude that $p_{j} \geq p_{j}^{M} \forall j \in\{A, B\}$ in any equilibrium. Next, $c<\pi_{j} p_{j}^{M}$ implies that $\mathcal{I}_{j}$ can only differ from $\mathcal{O}_{j}\left(p_{j}\right)$ by a zero measure set in any equilibrium. Hence, just as in the two-stage game, the targeting strategy of $j$ is essentially pinned down by $\mathcal{O}_{j}\left(p_{j}\right)$ whenever $j$ chooses $p_{j} \geq p_{j}^{M}$. As a result, for any prices $\left(p_{A}, p_{B}\right)>\left(p_{A}^{M}, p_{B}^{M}\right)$ to be part of an equilibrium, condition (A.1) must be satisfied (i.e., no firm has an incentive to marginally lower its price and target a bit further). By the proof of Theorem 2, this contradicts the presumption that $c<\pi_{j}\left(p_{j}^{M}-t\right) \forall j \in\{A, B\}$. Thus, there cannot be an equilibrium where $p_{j}>p_{j}^{M} \forall j \in\{A, B\}$.

Next, we show that mass advertising cannot arise as part of a Nash equilibrium with unlimited attention.

Proposition B4 In the simultaneous pricing-targeting game with unlimited attention, there cannot be a Nash equilibrium with an overlapping targeting profile.

Proof Suppose that $\lambda\left(\mathcal{I}_{A} \cap \mathcal{I}_{B}\right)>0$ holds in an equilibrium. Then, at least one firm $j \in\{A, B\}$ must be targeting a positive measure set of consumers in $\mathcal{P}_{-j} \cap \mathcal{I}_{-j}$, which cannot be optimal given that consumers are fully attentive and targeting is costly. Thus, there cannot be an equilibrium with overlap. 


\section{B.4 Persuasive Advertising}

Previous research has sometimes considered that advertising may affect consumer tastes in a persuasive way. The respective literature is heterogeneous in what it means to "persuade", ranging from mostly abstract shifts of the demand curve to very specific types of preferential shocks ${ }^{4}$ It is conceivable that, with utility-enhancing advertising, a heavily advertised alternative becomes more likely to be chosen. As this yields a similar prediction to the salience model of Section 5, it may become hard to disentangle both theories on observational grounds $5^{5}$ Moreover, the question remains whether persuasive advertising and attention competition predict the same equilibrium outcome in the targeting context studied by this article ${ }^{6}$ We demonstrate, by means of a simple argument, why this probably is not the case.

Suppose that the maximal willingness-to-pay of the consumers for a firm $j, V_{j}$, can be influenced by advertising. Let all consumers be fully attentive, and assume that the ads of firm $j$ increase $V_{j}$ without affecting $V_{-j}$, and abstract from price competition. We now argue that such a model of "pure" persuasion would most likely not lead to intrusive targeting profiles in equilibrium. Suppose that both firms are endowed with similar persuasion technologies and perfect targeting abilities. Assume that, say, firm $B$ targets a prime consumer $i$ of firm $A$, and manages to increase this consumer's valuation from $V_{B}$ to $V_{B}^{\prime}$ as a consequence of its persuasive advertising. Given that $i$ belonged to the prime segment of firm $A$ ex ante, firm $A$ can secure the demand of consumer $i$ by choosing a persuasion intensity that yields the valuation $V_{A}^{\prime}=V_{B}^{\prime}$. That is, firm $A$ can effectively counter any intrusion by $B$ with the same (or possibly even a lower) persuasion intensity. But if firm $B$ sees no reasonable hope of capturing such a consumer,

\footnotetext{
${ }^{4}$ See Bagwell (2007) for a survey, and Stigler and Becker (1977) for a critique of such persuasion.

${ }^{5}$ Field studies on persuasion cannot resolve this identification problem, but recent evidence from voting in US elections suggest that the effects from "persuasive" political ads are not long lasting (the effects wear off within a week) and exposure to ads matters (Gerber et al., 2011). Both observations seem more consistent with limited working memory and attention competition than with changes of the underlying preferences.

${ }^{6}$ We are grateful to a referee who inspired us to consider persuasive advertising within our targeting model.
} 
it will be reluctant to waste any resources to target the prime segment of $A$. It follows that the segmenting equilibrium is the likely outcome of such a game (also see Egli, 2015), in contrast to the mass advertising prediction in case of attention competition.

\section{References}

Armstrong, M. (2015). Search and ripoff externalities. Review of Industrial Organization 47, 273-302.

Bagwell, K. (2007). The economic analysis of advertising. In M. Armstrong and R. Porter (Eds.), Handbook of Industrial Organization, Volume 3. Amsterdan: Elsevier B.V.

Egli, A. (2015). Targeting and persuasive advertising. Mimeo.

Gerber, A. S., J. G. Gimpel, D. P. Green, and D. R. Shaw (2011). How large and long-lasting are the persuasive effects of televised campaign ads? results from a randomized field experiment. American Political Science Review 105, 135-150.

Stigler, G. J. and G. S. Becker (1977). De gustibus non est disputandum. American Economic Review 67, 76-90. 